\title{
Emergence of a tracer source from air concentration measurements, a new strategy for linear assimilation
}

\author{
J.-P. Issartel \\ Centre d'Enseignement et de Recherche en Environnement Atmosphérique, unité mixte Ecole Nationale des Ponts et \\ Chaussées - Electricité de France, France \\ Projet CLIME, équipe mixte Institut National de Recherche en Informatique et en Automatique - Ecole Nationale des Ponts et \\ Chaussées, France
}

Received: 30 March 2004 - Published in Atmos. Chem. Phys. Discuss.: 12 May 2004

Revised: 23 December 2004 - Accepted: 24 January 2005 - Published: 2 February 2005

\begin{abstract}
The measurement of atmospheric concentrations by a monitoring network is a promising tool for the identification of the widespread sources of trace species. The paper addresses the case of the species scattered linearly by a known meteorology. The question is classical: what can be said about the source from a set of measurements? Is it possible to guess from the values observed by the measurements that the source is spread close to the detectors, or that the tracer comes from a remote region? And, if the source was a point source, would it be possible to understand it by just considering these values? A part of the answers is a matter of practical sense: the resolution with which an emission can be retrieved will always be limited and probably lower for a remote region, even if the detectors and dispersion model are error free. The paper proposes a linear strategy of inference: to any set of values taken by the observed concentrations is associated linearly an estimate of the source. Doubled values lead to a doubled estimate. The method, based on adjoint techniques, is intended to optimise the resolution by quantifying, with the concept of illumination, which regions are well, poorly or not seen at all. The illumination tied to ordinary adjoint functions becomes excessive close to the detectors thus leading to inversion artefacts. This may be corrected by attributing each point of the space time domain a geometric and statistical weight. The adjoint functions are transformed. The choice of this renormalising function is constrained by an unambiguous entropic criterion preventing any overestimation of the available information that would lead to artefacts. It amounts to evenly distribute the information between the points organised with their weights as a "known domain". The theory is illustrated by calculations performed with the experimental source ETEX1.
\end{abstract}

Correspondence to: J.-P. Issartel

(issartel@cerea.enpc.fr)

\section{Introduction}

The atmospheric transport of tracers, especially the linear ones, is an active domain (Gallardo et al., 2002; Clerbaux et al., 2003; Baklanov and Mahura, 2004) with many investigations about the inverse problems (Bousquet et al., 2000; Rödenbeck et al., 2003). The natural interpretation of a measurement goes through its adjoint function (Marchuk, 1992; Robertson and Persson, 1993; Penenko and Baklanov, 2001). When a tracer is known to originate from an unknown point source, generally in an accidental context, the localisation of this origin has been widely studied (Sharan et al., 1995; Pudykiewicz, 1998; Seibert, 2001; Roussel et al., 2002; Issartel and Baverel, 2003; Penenko et al., 2002). The reconstruction of the widespread sources of such species as carbon dioxide, monoxide or methane is a more general and very different inverse problem. It involves adjoint techniques (Marchuk, 1964; Tarantola, 1987; Uliasz and Pielke, 1991; Enting et al., 1995; Enting, 2000; Wotawa et al., 2003). At the moment of the measurement the history of the sample is concentrated in the detector. This results in singularities (Ashbaugh et al., 1985; Stohl, 1998; Baklanov, 2000) holding the reconstruction of the source up. This corresponds to the classical problem of data assimilation: how spreading the local information to the whole system (Bouttier and Courtier, 1999). The obstacle compromises the achievement of the natural idea, based on the perturbation theory (Gram, 1879; Cheney, 1966; Marchuk, 1973), to use as base functions of the inverse problem the adjoint concentrations of the measurements. Bennett and McIntosh (1982) have proposed to reduce the singular influence of the detectors by a system of weights. This idea is taken again here through the concept of illumination (Sect. 2). The latter was introduced in (Issartel, 2003) to give the singularities a sense. The, influence of the detectors could then be efficiently controlled by

(C) 2005 Author(s). This work is licensed under a Creative Commons License. 
means of weights empirically adjusted to the various parts, in space and time, of the domain under investigation. The present paper aims at understanding the geometric and statistical meaning of the weights and put their choice on non empirical bases.

The method proposed hereafter (summarised in the Sect. 3) is intended to improve the source estimation in general. Its optimality is that of a compromise: not all possible sources will be restored equally well. The question behind the work is the following. The values $\mu_{1}, \ldots, \mu_{n}$ have been obtained from concentration measurements at various positions and dates, the meteorological fields are known, there exists no other piece of information, even indirectly, except perhaps the positivity of the source: what best can be said ? In particular, if a point source has been used to prepare artificial values of the measurements, it is hoped that the inversion procedure will 'understand' the source is a point by just considering the special set of values of $\mu_{1}, \ldots, \mu_{n}$. This aim of an infinite resolution in space and time is clearly an impossible wish with a few tenths, or thousands, of measurements. The details will be necessarily smoothed out for lack of information. A point will become a smaller or greater spot scattering in space and time with a shape determined by the current winds and diffusion. This smoothness of the estimation corresponds to a physical limit. Some calculations show that the smoothing expected from the finite resolution of the current meteorological models is not satisfactory. The use of the weights enables to explore this limit. In fact they transform the geometry of the space time domain with a new metrics, a new weighted scalar product and new adjoint concentrations (Sect. 5). The weighted geometry may as well be interpreted with the introduction of virtual statistics (Sect. 6), hereafter called anticipations, describing the outcome expected from the measurements. Then, based on the idea that the part of a source not seen by the inversion, somehow, does not exist, the weighted product is shown physically equivalent to a fuzzy product no longer seeing points. The concept of illumination will enable to optimally adjust the weights according to an entropic criterion preventing from an abusive and artificial use of the information (Sect. 7).

The quality of the estimation is limited through the smoothness and fuzziness by the availability of the information, it is also limited by the measurement errors due to the technical performance of the detectors and to the representativity of the dispersion model. There the present theory drifts significantly from the usual background of assimilation as the two limitations are considered fundamentally different and independent. The smoothness and fuzziness limitation with the statistics of the anticipations have nothing to do with a classical error (Sect. 8). The fuzzy version of the weighted product seems to compare well with the background error covariance matrix of the classical theory as both provide a local geometric description of the regions well or poorly seen. Nevertheless the first one is calculated directly from the meteorological conditions, position and dates of the measurements independently of their effective values. The second one is related to a model of the source with an a priori evaluation amended a posteriori once the values of the measurements are known. The equations of the estimate turn out to be different. In particular the compromise of the classical cost function between a source model and the observations becomes irrelevant. In compensation a new variable, the informational energy, may be defined to foresee the good or poor identifiability of a source (Sect. 9). This new quantity is shown to be pivotal in the interpretation of the estimate, with perhaps a greater relevance than the estimate itself. It seems also that, through the informational energy, bridges open towards theoretical physics. The geometry of the present theory is summarised by defining a "known domain" where the information is evenly distributed (Sect. 10). Finally (Sect. 11) the strategy is compared to the first ETEX experiment with synthetic and real data. ETEX1 was a twelve hour point release (Brandt et al., 1997; Wendum, 1998; Seibert, 2001). Nevertheless the method is primarily devoted to the identification of widespread sources. Thus the case of a Gaussian distribution in space and time was investigated based on synthetic measurements. Synthetic measurements were used as well to explore the behaviour of the method when a point source is very close to some detector. This emphasises the requirement that the monitoring network should be designed consistently with the proper geometry and smoothness of the sources to observe. The notations used throughout the paper are summarised in the Table 1 where the physical units are given, different if the sought source is spread in the volume of the atmosphere $\Omega$ (case 1) or at the surface of the ground $\Sigma$ (case 2). The many scalar products used in the paper are listed there.

\section{Reminder about the illumination}

The mixing ratio of an atmospheric tracer in unit amount of tracer per unit mass of air will be denoted $\chi(\boldsymbol{x})$ with a space-time coordinate $\boldsymbol{x}=(x, y, z, t)$. In $\Omega$, the atmospheric domain, $(x, y)$ is the horizontal position and $z$ the altitude; $\mathrm{t}$ is the date in the time domain $\mathrm{T}$. Under investigation is the source $\sigma(x, y, z, t)=\sigma(\boldsymbol{x})$ of this tracer in unit amount of tracer per unit mass of air and per unit time. We shall suppose that there is a linear link $\chi=\mathcal{L}(\sigma)$ corresponding to the following dispersion law including a turbulent term $\zeta(\chi)$ and a term for linear creation or killing $\alpha \chi$ describing for instance a radioactive decay or a position dependent scavenging rate $\alpha(\boldsymbol{x})$. The law must be adequately complemented by zero boundary conditions. The wind is denoted $\boldsymbol{v}(\boldsymbol{x})$ :

$$
\frac{\partial \chi}{\partial t}+\boldsymbol{v} \cdot \nabla \chi+\zeta(\chi)+\alpha \chi=\sigma
$$

The concentration measurements $\mu_{1}, . ., \mu_{n}$ used to infer $\sigma$ may be described in a very symmetric way. To describe the 
Table 1. Definition of the variables (uat: unit amount of tracer)

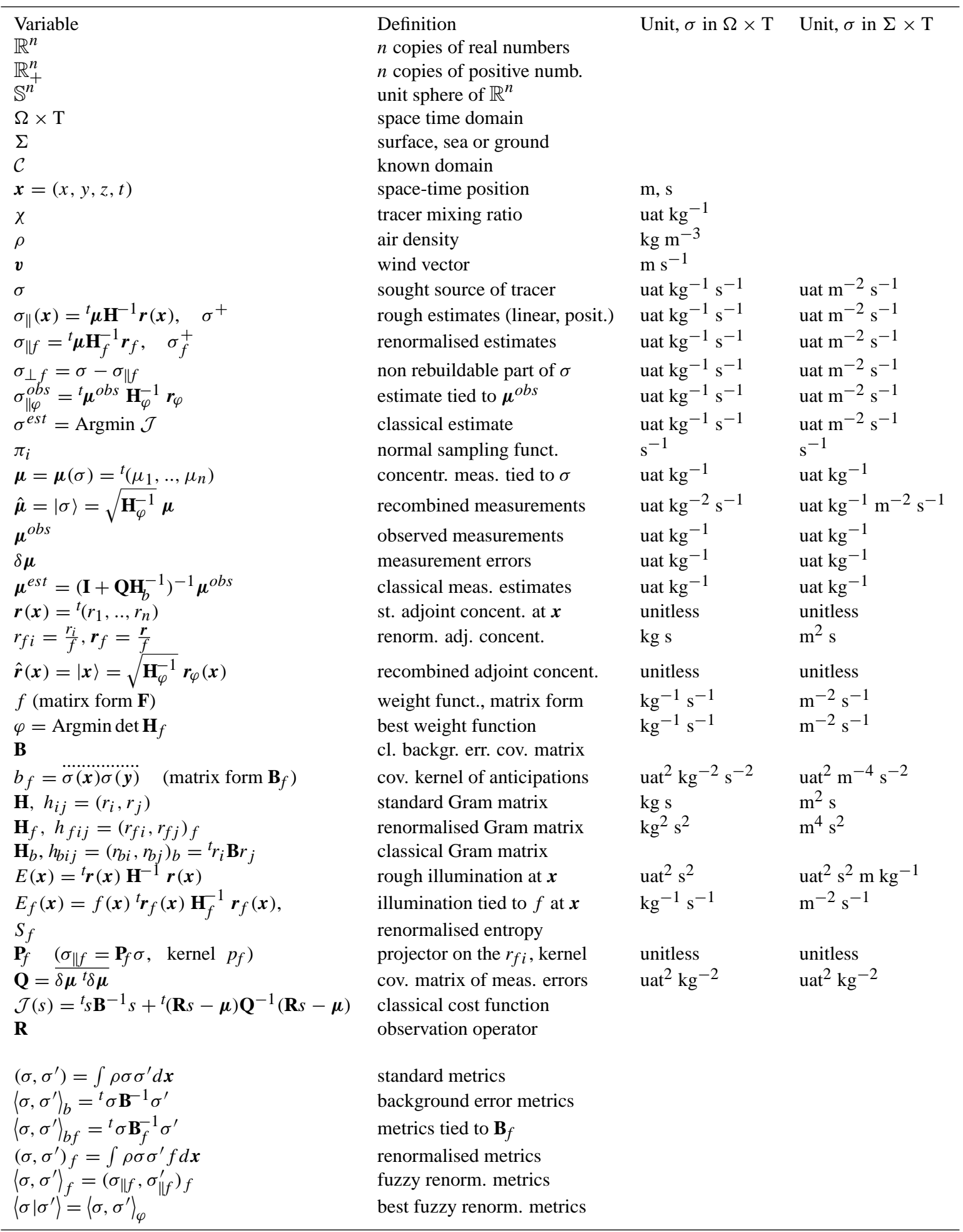


6 stations, 51 measurements

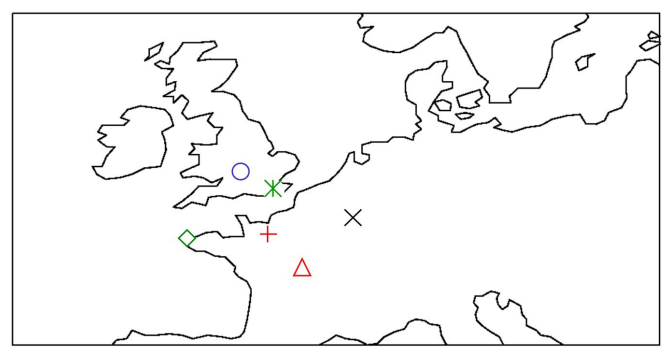

15 stations, 137 measurements

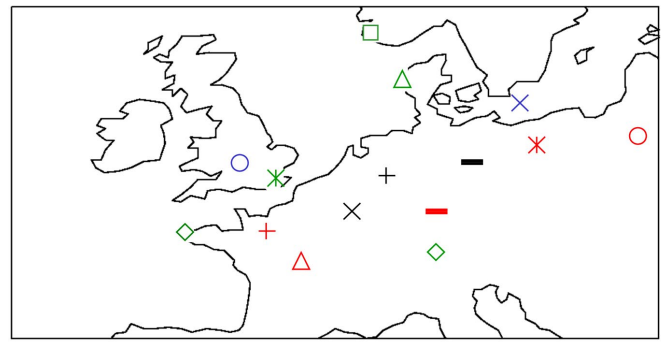

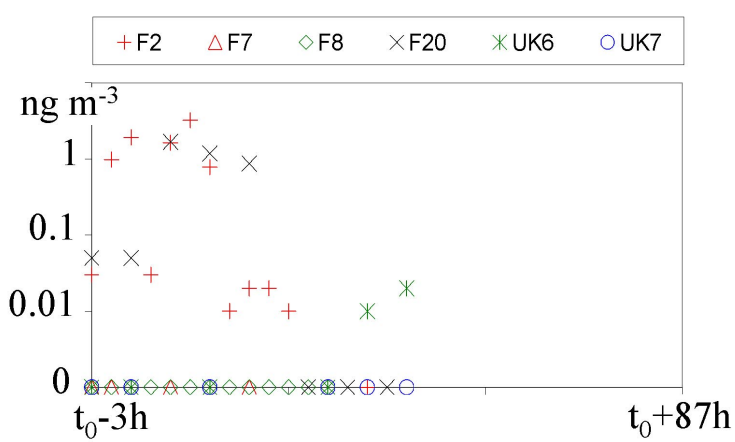

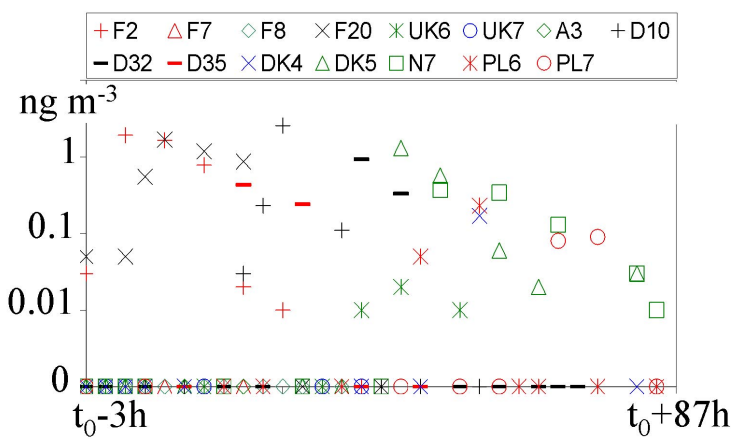

Fig. 1. Geographic and time distribution of the two selections of measurements used for the calculations: upper two images, the 51 measurement selection, lower two images the 137 measurement selection. The time $t_{0}$ corresponds to the beginning of the pmch release in Monterfil, 23 Oct. 1994, 16 UT; the first sampling period begins at $t_{0}-3 h$. Different detectors are indicated by different symbols. The values in ng of pmch per $\mathrm{m}^{3}$ are from the ETEX1 database. The ng. $\mathrm{m}^{-3}$ are indicated on a logarithmic scale except that all values less than 0.001 have been represented as zero.

sample number $i$ we need know how much air has been taken where and when; a function $\pi_{i}(\boldsymbol{x})$ is introduced:

$\mu_{i}=\int_{\Omega \times T} \rho \chi \pi_{i}(\boldsymbol{x}) d \boldsymbol{x}$

The unit of the $\pi_{i}$ is tied to that of the $\mu_{i}$. Here with the $\mu_{i}$ described as mixing ratios, the $\pi_{i}$ will be normalised with respect to the mass of the samples: $\int \rho \pi_{i}(\boldsymbol{x}) d \boldsymbol{x}=1$. The measurements behave like a scalar product:

$\mu_{i}=\left(\chi, \pi_{i}\right)=\left(\mathcal{L}(\sigma), \pi_{i}\right) \quad(\phi, \psi)=\int_{\Omega \times T} \rho \phi \psi d x$

By introducing the adjoint operator $\mathcal{L}^{*}$ and the adjoint concentrations $r_{i}=\mathcal{L}^{*}\left(\pi_{i}\right)$ we obtain:

$\mu_{i}=\int_{\Omega \times T} \rho \sigma r_{i} d \boldsymbol{x}, \quad-\frac{\partial r_{i}}{\partial t}-\boldsymbol{v} \cdot \nabla r_{i}+\zeta\left(r_{i}\right)+\alpha r_{i}=\pi_{i}$

The derivation of the adjoint law for $r_{i}$ is described in (Issartel and Baverel, 2003) with emphasis to the self adjoint nature of $\zeta$ in the case of a time-symmetric turbulence; the adjoint law must be complemented by the adjoint boundary conditions which are in fact zero boundary conditions. A linear combination $\sigma_{\|}$of the $r_{i}$ might be proposed as an estimation for $\sigma$. The coefficients would be obtained linearly from the measurements after inverting the Gram covariance matrix $\mathbf{H}$ of the $r_{i}$ :

$\boldsymbol{\mu}=\left[\begin{array}{c}\mu_{1} \\ : \\ \mu_{n}\end{array}\right] \quad \boldsymbol{\lambda}=\left[\begin{array}{c}\lambda_{1} \\ : \\ \lambda_{n}\end{array}\right] \quad \mathbf{H}=\left[h_{i, j}\right] \quad h_{i, j}=\left(r_{i}, r_{j}\right)$

$\sigma_{\|}(\boldsymbol{x})=\sum_{i=1}^{n} \lambda_{i} r_{i}(\boldsymbol{x}) \quad$ with $\quad \boldsymbol{\lambda}=\mathbf{H}^{-1} \boldsymbol{\mu}$

We shall use as well the following notations:

$\sigma_{\|}={ }^{t} \lambda \cdot \boldsymbol{r} \quad$ with $\quad \boldsymbol{r}(\boldsymbol{x})=\left[\begin{array}{c}r_{1}(\boldsymbol{x}) \\ : \\ r_{n}(\boldsymbol{x})\end{array}\right] \in \mathbb{R}_{+}^{n}$

As noticed in (Issartel, 2003) this strategy is not well defined. Compared to the atmosphere the samples are very small so that the $r_{i}$ display peaks by the position of the detectors. The self interaction coefficients $h_{i, i}$ are very large; they are singular in the theoretical case of Dirac detectors. Calculations with various resolutions show, on the Figs. 2, 3, 10, 11, that the effect of the singularity persists even when the detectors are modelled as large as one mesh. This failure was described 

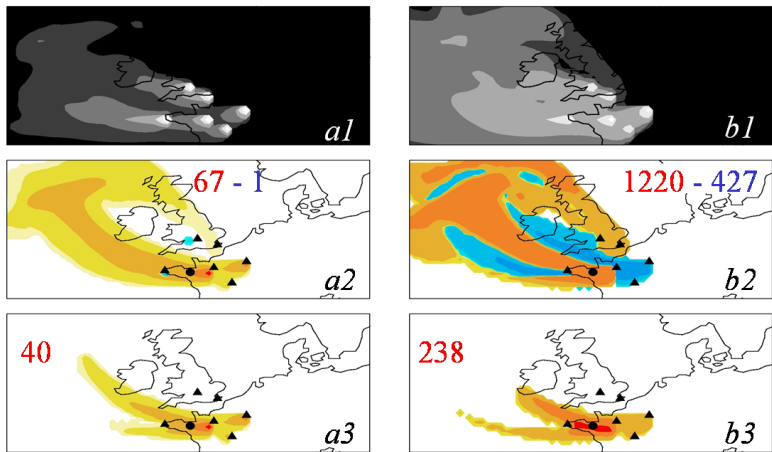

$b 3$ $\mathrm{km}^{-2}$
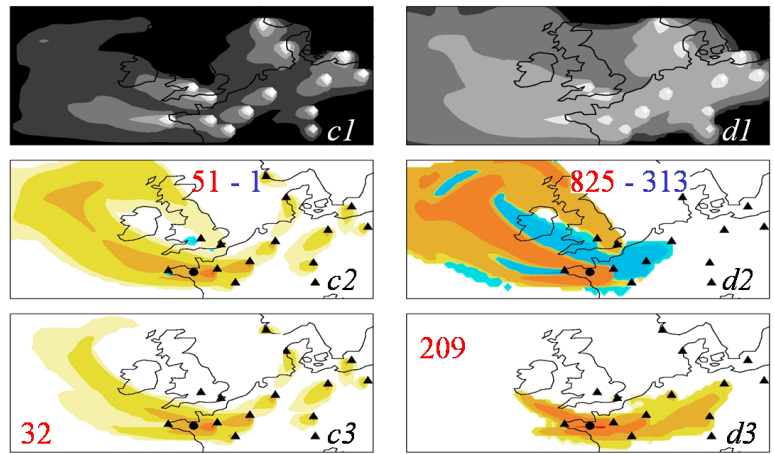

209

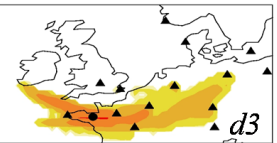

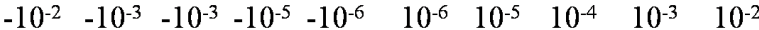

Fig. 2. The figure represents the illumination on the first line, the source rebuilt for the ETEX release as a linear combination of the adjoint functions on the second line, under positivity constraint on the third line; these variables are integrated in time for the whole duration of the calculation performed with a horizontal resolution of $0.5^{\circ} \times 0.5^{\circ}$. Synthetic values were used for the measurements, 51 in (a) and (b), 137 in (c) and (d). No correction of the illumination is made in $a$ nor in $c$. The optimal renormalisation was performed for (b) and (d). The red and blue numbers indicate the total amount in $\mathrm{kg}$ of the positive and negative releases respectively. The release is indicated by a dot and the detectors by triangles.
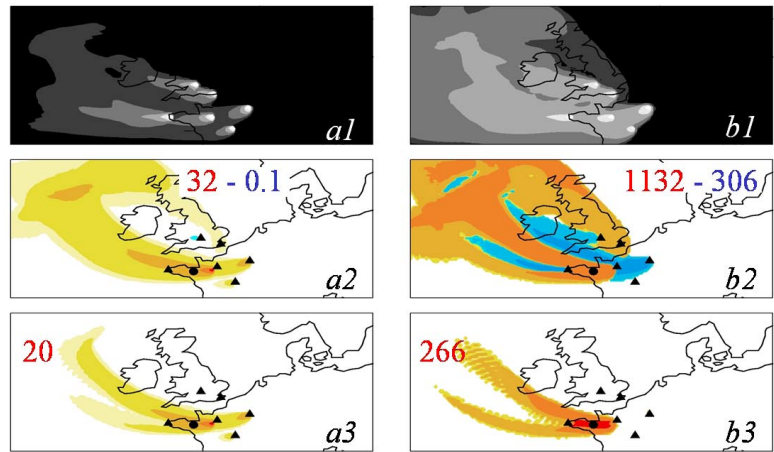

$b 3$

\begin{tabular}{lllll}
$10^{-7}$ & $10^{-6}$ & $10^{-5}$ & $10^{-4}$ & $10^{-3}$ \\
\hline
\end{tabular} $\mathrm{km}^{-2}$
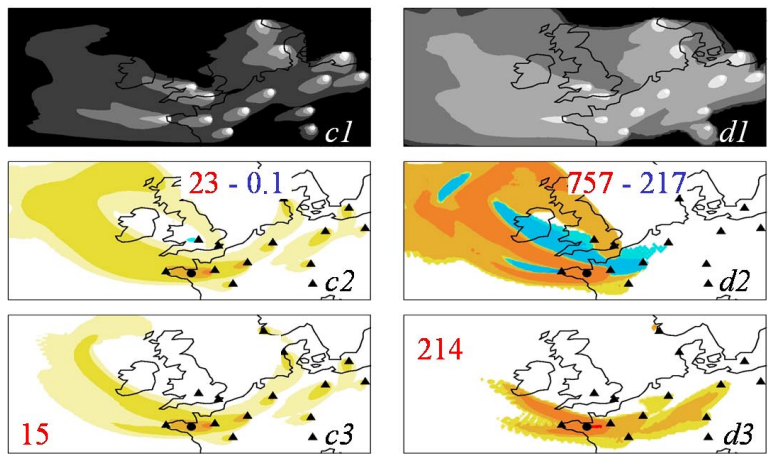

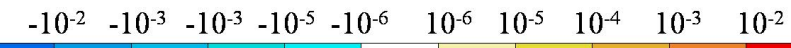
$3.4 \mathrm{~kg} \cdot \mathrm{km}^{-2}$

Fig. 3. The figure represents the same calculations as the Fig. 2 now performed with a higher horizontal resolution of $0.25^{\circ} \times 0.25^{\circ}$. The synthetic values used for the inversion are nevertheless those produced by the previous calculation. Thus the same list of measurement values have been inverted. Accordingly the figures differ only for the inversion which includes the calculation of the adjoint retroplumes.

in terms of a function of illumination $E$; the left superscript $t$ stands for the transposition:

$$
E(\boldsymbol{x})={ }^{t} \boldsymbol{r}(\boldsymbol{x}) \mathbf{H}^{-1} \boldsymbol{r}(\boldsymbol{x}) \geq 0, \quad \int_{\Omega \times \mathrm{T}} \rho E(\boldsymbol{x}) d \boldsymbol{x}=n
$$

As $\mathbf{H}$ is a Gram matrix, it is positive definite and so is $\mathbf{H}^{-1}$. The second equation is the trace of the definition 5 of $\mathbf{H}$ : $\int \rho \mathbf{H}^{-1} \boldsymbol{r}^{t} \boldsymbol{r} d \boldsymbol{x}=\mathbf{I} ; \mathbf{I}$ is the $n \times n$ identity matrix. The matrix $\mathbf{M}(\boldsymbol{x})=\mathbf{H}^{-1} \boldsymbol{r}(\boldsymbol{x})^{t} \boldsymbol{r}(\boldsymbol{x})$ is the influence the inversion attributes to a unit mass of ambient air around $x$ : the contribution from this point to measurements $\boldsymbol{\mu}$ is estimated as $\rho \mathbf{M}(\boldsymbol{x}) \boldsymbol{\mu} d \boldsymbol{x}$. The illumination $E(\boldsymbol{x})$ is the trace of $\mathbf{M}(\boldsymbol{x})$ which enables to interpret it as the share attributed to $x$ of the $n$ available pieces of information. For a point seen by no detector $E(\boldsymbol{x})$ vanishes; in the neighbourhood of the detectors $E(\boldsymbol{x})$ becomes large, all the larger as the detectors are smaller. This bad geometry is transmitted to the estimate $\sigma_{\|}$as illustrated by Figs. 2, 3a, c. In the case of gridded calculations this geometry is finitely bad because the detectors, described by at least a complete mesh, are finitely small. As the detectors are much smaller a resolution refinement causes the illumination, and the source estimate, to more and more focus around them. In the limit case of infinitely small Dirac detectors the estimate reduces to infinitesimal sources inside the detectors as nothing else is seen. This behaviour is illustrated by the Fig. 3a, c compared to the Fig. 2a, c. 

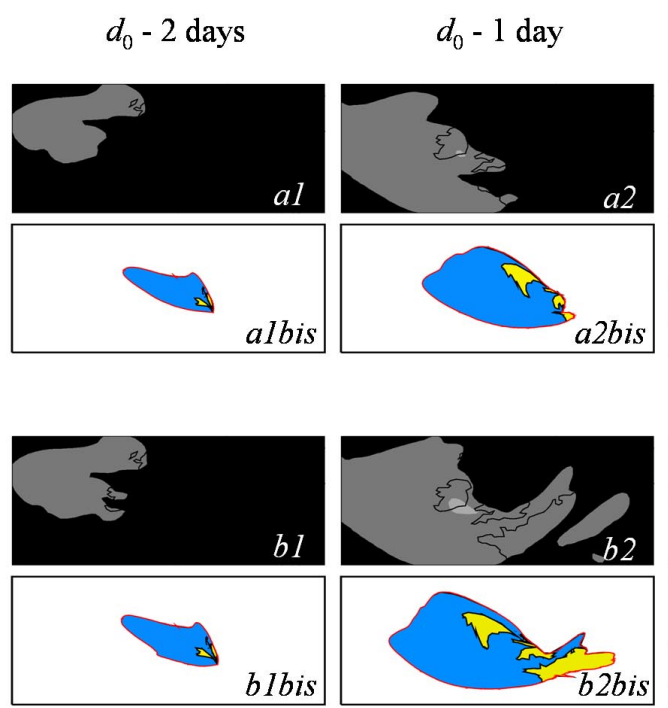

$\begin{array}{llll}10^{-6} & 10^{-5} & 10^{-4} & 10^{-3} \\ \mathrm{~km}^{-2} \mathrm{day}^{-1}\end{array}$ $d_{0}:$ Oct. 1994 23/10UT - 24/10UT
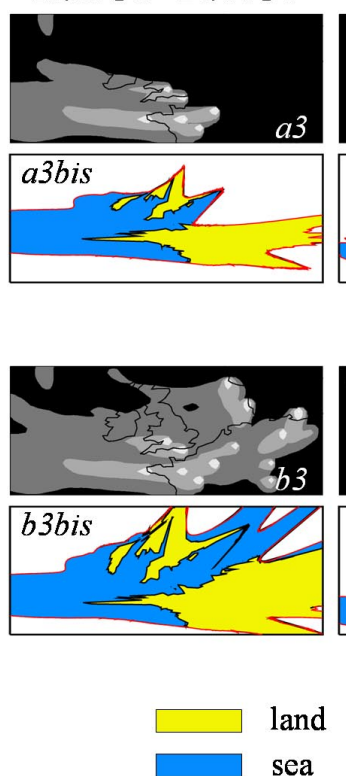

$d_{0}+1$ day
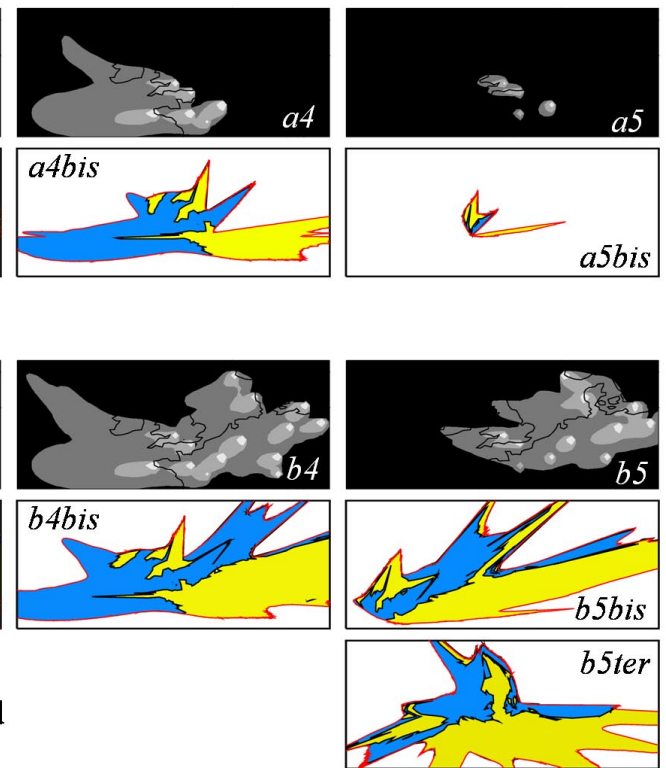

Fig. 4. Evolution of the renormalised illumination for the selections of 51 measurements in (a), 137 in (b). The illumination has been integrated in time for five successive $24 \mathrm{~h}$ periods, the third one beginning 6 hours before and finishing 6 hours after the ETEX release. On the figures bis this daily integrated illumination is transformed into a geometry by means of a radial transformation centred at Monterfil.The figure b5ter was obtained with a centre of the radial transformation in the Danish islands. The distortions are less in b5ter than in b5bis but the areas of the various regions are the same on both figures.

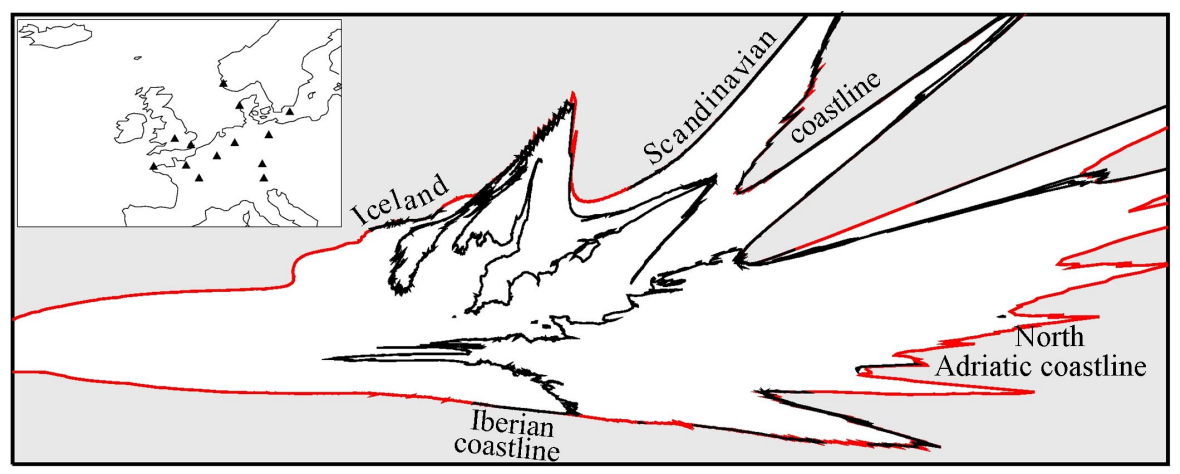

Fig. 5. Enlargement of the Fig. 4 b3bis with emphasis to the coastlines in black, especially close to the horizon in red. The regularisation of the coasts has not been activated in order to better show the increasing instability of the calculation close to the horizon. In the subfigure the triangles indicate the detectors operated during the $24 \mathrm{~h}$ period (see Fig. 4).

We proposed in (Issartel, 2003) to smooth the inversion by introducing a renormalising function $f(x) \geq 0$ and a new product $(,)_{f}$ :

$\left(\sigma, \sigma^{\prime}\right)_{f}=\int_{\Omega \times \mathrm{T}} \rho(\boldsymbol{x}) f(\boldsymbol{x}) \sigma(\boldsymbol{x}) \sigma^{\prime}(\boldsymbol{x}) d \boldsymbol{x}$

$\mu_{i}=\int \rho f \sigma(\boldsymbol{x}) r_{f i} d \boldsymbol{x}=\left(\sigma, r_{f i}\right)_{f} \quad r_{f i}(\boldsymbol{x})=\frac{r_{i}(\boldsymbol{x})}{f(\boldsymbol{x})}$
The estimate now becomes $\sigma_{\| f}$ :

$$
\begin{array}{lc}
\mathbf{H}_{f}=\left[h_{f, i, j}\right] & h_{f, i, j}=\left(r_{f i}, r_{f j}\right)_{f}=\int_{\Omega \times \mathrm{T}} \rho \frac{r_{i} r_{j}}{f} d \boldsymbol{x} \\
\lambda_{f}=\mathbf{H}_{f}^{-1} \boldsymbol{\mu} & \sigma_{\| f}(\boldsymbol{x})={ }^{t} \boldsymbol{\lambda}_{f} \cdot \boldsymbol{r}_{f}(\boldsymbol{x})
\end{array}
$$

This approach removes the peaks from the $r_{f i}$. Indeed to $f$ and its scalar product are tied, through a modified version of 
$\begin{array}{cc}d_{0}-2 \text { days } & d_{0}: \text { oct. } 1994 \\ & d_{0}-1 \text { day } \\ 23 / 10 \mathrm{UT}-24 / 10 \mathrm{UT}\end{array}$

$d_{0}+1$ day

$d_{0}+2$ days
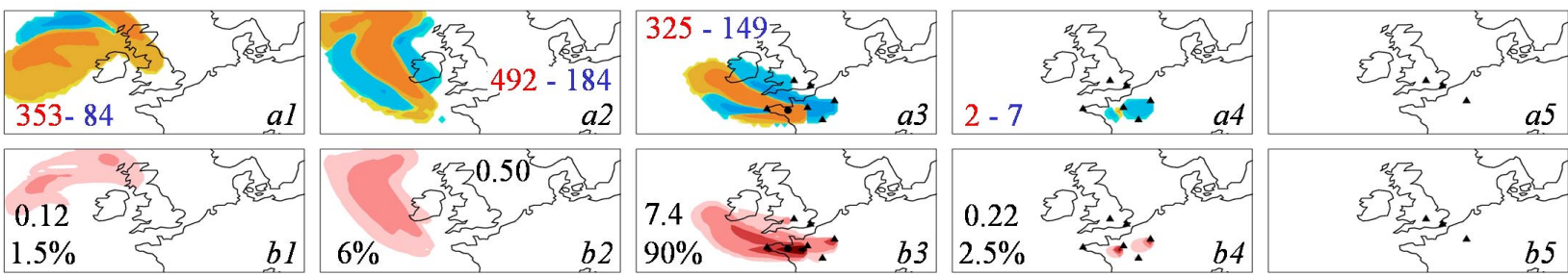

synthetic measurements
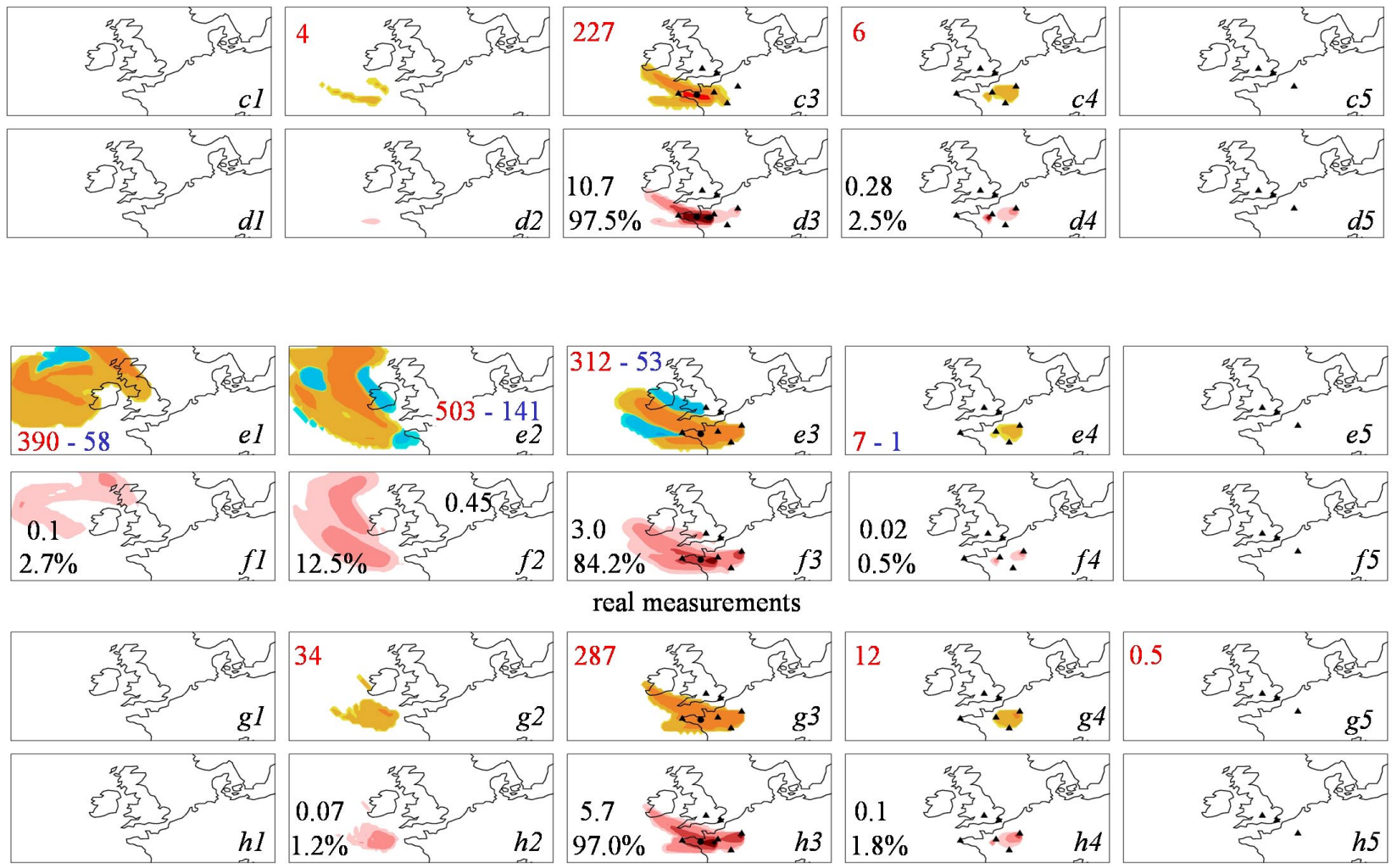

\begin{tabular}{|c|c|c|c|c|c|}
\hline$-10^{-2}$ & $-10^{-3}$ & $-10^{-4}-10^{-5}$ & $10^{-5}$ & $10^{-4}$ & $10^{-3}$ \\
\hline
\end{tabular}

$3.4 \mathrm{~kg} \mathrm{~km}^{-2}$ day ${ }^{-1}$

$\frac{10^{-12} 10^{-11} \quad 10^{-10} \quad 10^{-9} \quad 10^{-8}}{\left(3.4 \mathrm{~kg} \mathrm{~km}^{-2} \text { day }^{-1}\right)^{2} \mathrm{~km}^{-2} \text { day }^{-1}}$

Fig. 6. Daily integrated evolution of the source rebuilt for the ETEX release by means of renormalised adjoint functions. In (a) the source rebuilt linearly for synthetic measurements is integrated on $24 \mathrm{~h}$ periods, and in (c) the source rebuilt under positivity constraints. The red and blue numbers correspond to the total in $\mathrm{kg}$ of the positive and negative releases during the successive periods. The energy released by both estimate is integrated on the same periods respectively in (b) and (d). This amount is indicated for the five periods as an absolute value in $10^{-6}\left(3.4 \mathrm{~kg} \mathrm{~km}^{-2} \mathrm{day}^{-1}\right)^{2}$ and as a percentage of the total energy release of the estimate. The dot and the triangles indicate the ETEX release and the detectors when they are active. The figures $(\mathbf{e}),(\mathbf{f}),(\mathbf{g}),(\mathbf{h})$ are obtained similarly for the real values from the ETEX1 database.

the Eq. 7, a renormalised illumination $E_{f}$ :

$$
\begin{aligned}
& E_{f}(\boldsymbol{x})=f(\boldsymbol{x}){ }^{t} \boldsymbol{r}_{f}(\boldsymbol{x}) \mathbf{H}_{f}^{-1} \boldsymbol{r}_{f}(\boldsymbol{x}) \\
& \int_{\Omega \times \mathrm{T}} \rho{ }^{t} \boldsymbol{r}_{f}(\boldsymbol{x}) \mathbf{H}_{f}^{-1} \boldsymbol{r}_{f}(\boldsymbol{x}) d \boldsymbol{x}=n
\end{aligned}
$$

The inversion was clearly improved for the following empirical choice $f(\boldsymbol{x})=\max \left[E(\boldsymbol{x}), \frac{E_{\max }}{1000}\right]$ inferred from the observation that around the detectors the illumination $E$ decreased a factor 1000 from its maximum value for irrelevant space scales. The aim of the present paper is to put the choice of the renormalising function on non empirical bases. 

$d_{0}-2$ days
$d_{0}$ - 1 day
$d_{0}:$ oct. 1994
23/10UT - 24/10UT
$d_{0}+1$ day
$d_{0}+2$ days
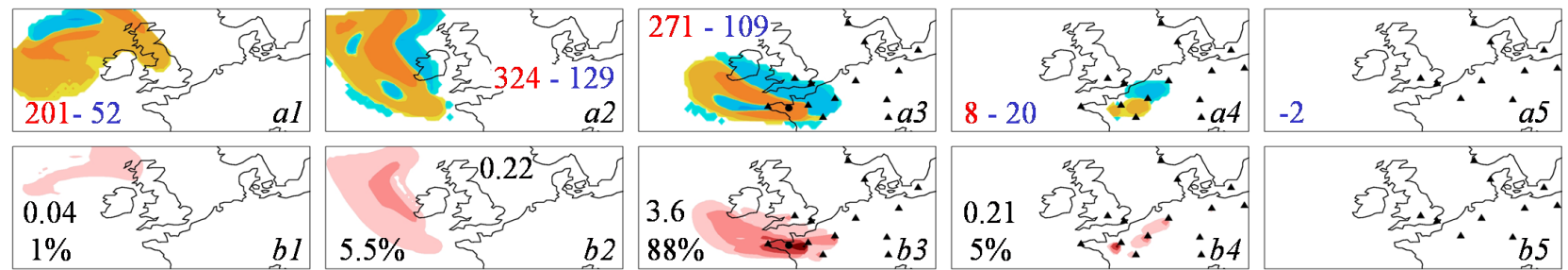

synthetic measurements
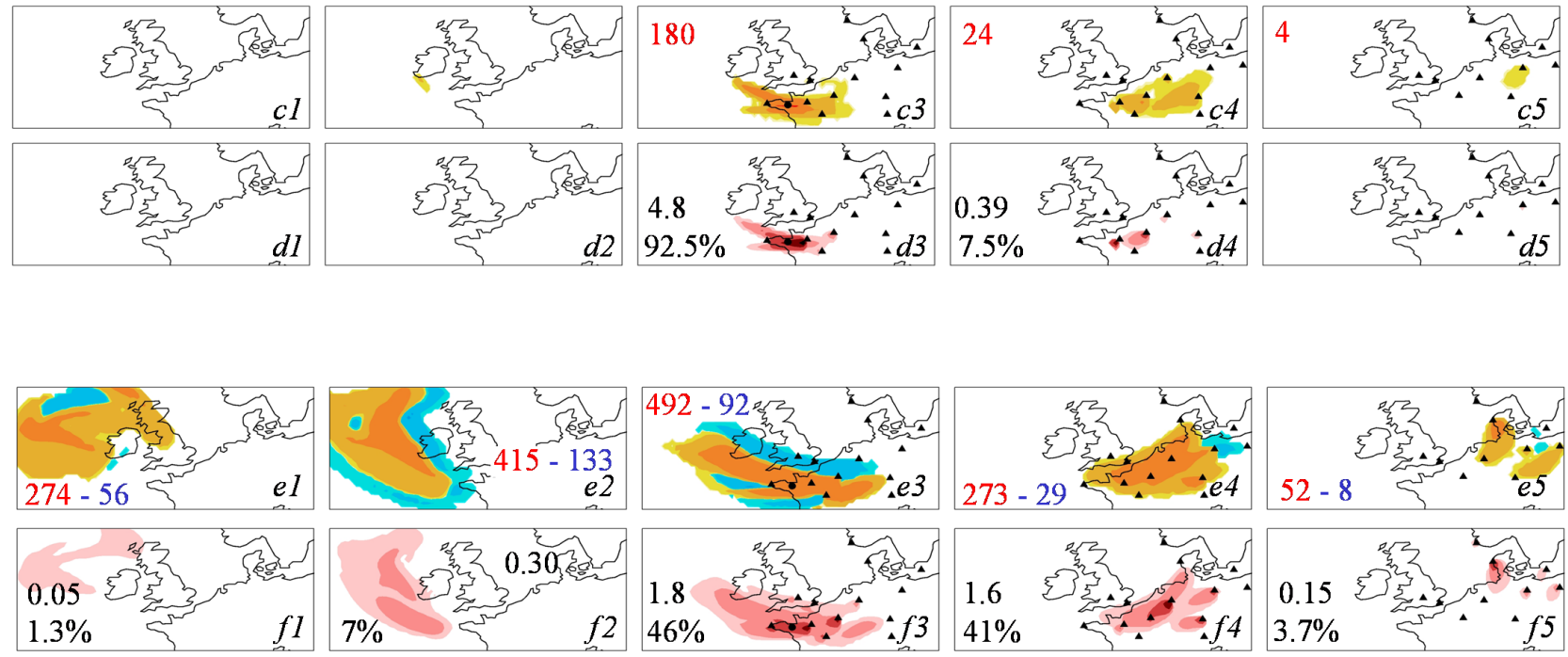

real measurements
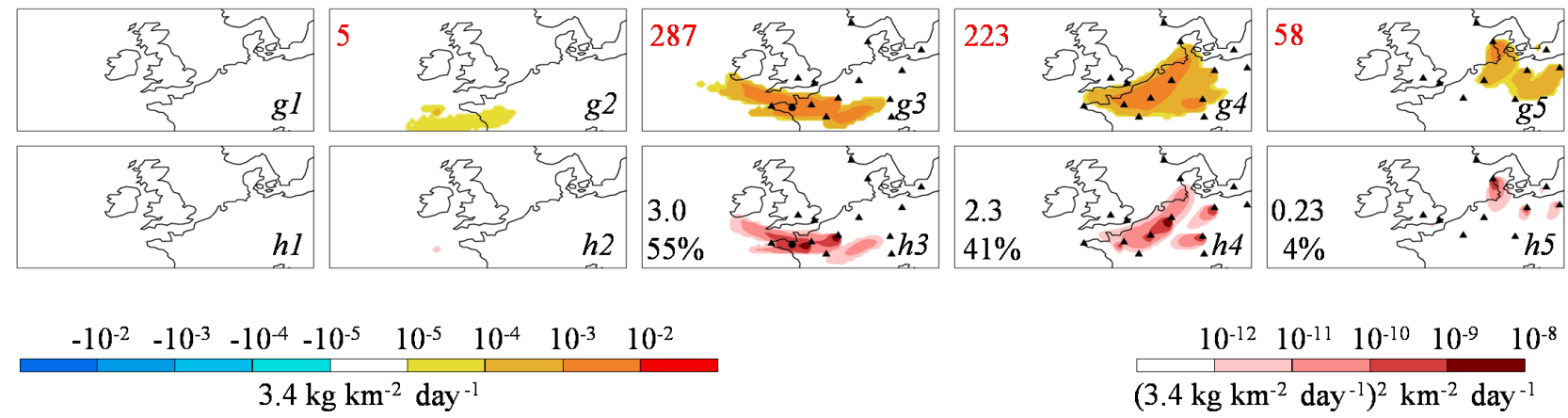

Fig. 7. Results obtained for the selection of 137 measurements. The organisation of this figure is parallel to that of the Fig. 6 .

\section{Practical calculation of the estimate}

This section describes the operations performed to reach our estimates starting from the observations $\boldsymbol{\mu}$. The algebraic estimate is linear with respect to the measurements. The positive one associated non linearly to a set of non negative measurements is relevant when the sought source is known to be purely non negative. The theoretical justifications will be addressed mainly in the subsequent sections.
The first step is the preliminary calculation of the adjoint functions $r_{i}$. As passive tracers are considered the adjoint Eq. (4) is just a retrograde version of the forward advection diffusion law 1 and $\alpha=0$. No adjoint model is required: the forward model is handled with a backward reading of the meteorological archives, the sign of the winds is changed, the sign of the diffusion (and decay coefficient if any) is not. The detector is generally modelled as sampling one complete grid mesh. The accurate computation of the high values of the $r_{i}$ inside the detectors is all the more avoided as the resolution is low. 
The second step is the determination of the optimal renormalising function that will be denoted $\varphi$. As will be seen, $\varphi$ is completely characterised by the following equation valid for all $\boldsymbol{x}$ effectively seen:

$\boldsymbol{r}(\boldsymbol{x}) \neq 0 \Longrightarrow{ }^{t} \boldsymbol{r}_{\varphi}(\boldsymbol{x}) \mathbf{H}_{\varphi}^{-1} \boldsymbol{r}_{\varphi}(\boldsymbol{x})=1$

This equation means that in the geometry weighted by $\varphi$, the illumination becomes homogeneous, all points become equally well seen. Despite its forbidding appearance this equation is easily solved by a few iterations of the following empiric algorithm. We did not demonstrate mathematically its observed efficiency. The iterative procedure may be initiated with $f_{0}(\boldsymbol{x}) \equiv 1$ corresponding to the ordinary geometry associated to the illumination ${ }^{t} \boldsymbol{r}(\boldsymbol{x}) \mathbf{H}^{-1} \boldsymbol{r}(\boldsymbol{x})$. In fact, the following problem is solved:

${ }^{t} \boldsymbol{r}(\boldsymbol{x}) \mathbf{H}^{-1} \boldsymbol{r}(\boldsymbol{x}) \geq \beta \quad \Longrightarrow{ }^{t} \boldsymbol{r}_{\varphi}(\boldsymbol{x}) \mathbf{H}_{\varphi}^{-1} \boldsymbol{r}_{\varphi}(\boldsymbol{x}) \simeq 1$

The algorithm works well provided to stand sufficiently aside, with $\beta$, from the points not seen at all where $\boldsymbol{r}(\boldsymbol{x})=0$. The iterations are defined on the selected domain by:

$f_{k+1}(\boldsymbol{x})=f_{k}(\boldsymbol{x}) \sqrt{{ }^{{ }_{\boldsymbol{r}_{f}}}(\boldsymbol{x}) \mathbf{H}_{f_{k}}^{-1} \boldsymbol{r}_{f_{k}}(\boldsymbol{x})}$

For the calculations presented here no more than nine iterations led to $0.985 \leq{ }^{t} \boldsymbol{r}_{f_{9}} \mathbf{H}_{f_{9}}^{-1} \boldsymbol{r}_{f_{9}} \leq 0.995$.

The third step is the calculation of the transformed adjoint functions $r_{\varphi i}(\boldsymbol{x})=\frac{r_{i}(\boldsymbol{x})}{\varphi(\boldsymbol{x})}$. The points poorly seen are discarded by simply considering $r_{\varphi i}(\boldsymbol{x})=0$. The influence of the cutting parameter $\beta$ illustrated by the Fig. 12 will be discussed later.

The fourth step is simply the calculation of the estimate

$\lambda_{\varphi}=\mathbf{H}_{\varphi}^{-1} \boldsymbol{\mu} \quad \sigma_{\| \varphi}(\boldsymbol{x})={ }^{t} \boldsymbol{\lambda}_{\varphi} \cdot \boldsymbol{r}_{\varphi}(\boldsymbol{x})$

A fifth step may be added when the sought source is known to be everywhere non negative. Even if the measurements are all positive $\sigma_{\| \varphi}(\boldsymbol{x})$ may be locally negative. A positive estimate $\sigma_{\varphi}^{+}$can be calculated according to the method described in (Issartel, 2003). The reader is reminded that $\sigma_{\| \varphi}(\boldsymbol{x})$ minimises the norm $\left(\sigma_{\| \varphi}, \sigma_{\| \varphi}\right)_{\varphi}$ under the constraint that the measurements are satisfied. The positive estimate has a minimal norm $\left(\sigma_{\varphi}^{+}, \sigma_{\varphi}^{+}\right)_{\varphi}$ under the additional constraint to be everywhere non negative. Its calculation is based on the conjecture, symbolised below by the question mark, that:

$\sigma_{\| \varphi}(\boldsymbol{x}) \leq 0 \stackrel{?}{\Longrightarrow} \sigma_{\varphi}^{+}(\boldsymbol{x})=0, \quad \boldsymbol{x} \in \Omega \times \mathrm{T}$

Iterating from $\sigma_{\| \varphi}=\sigma_{\| \varphi(0)}$, estimates $\sigma_{\| \varphi(k)}$ are obtained by inverting matrices $\mathbf{H}_{\varphi(k)}$ with the same definition 9 as $\mathbf{H}_{\varphi}$ except that the domain of integration is a part of $\Omega \times \mathrm{T}$ shrinking to eliminate the negative parts of the successive estimates. After some tenths of iterations the result always successfully passes the necessary and sufficient optimality conditions of Karush, Kuhn and Tucker (Rockafellar, 1970).
Nevertheless the conjecture was proved false by J.-B. Baillon with a counter example reported in (Issartel, 2003).

The above operations imply the inversion of a number of symmetric positive definite matrices. The matrices $\mathbf{H}_{f_{k}}$ and $\mathbf{H}_{\varphi}$ successively considered in the second and third steps are usually well conditioned but the conditioning of the matrices $\mathbf{H}_{\varphi(k)}$ of the fifth step rapidly increases up to unmanageable values. The inversions were regularised by a Truncated Singular Value Decomposition (Bertero et al., 1985, 1988; Fan et al., 1999). Practically the inversion of the little eigenvalues was topped as reported in (Issartel, 2003). The estimates are finally directly sensitive only to the square root of the regularised conditioning which, accordingly, was chosen here as large as 900 .

The source of a pollutant emitted at the surface $\Sigma$ of the ground or oceans may be described as a release $\sigma_{s}(x, y, t)$ at the altitude $z_{s}(x, y)$ in unit amount of tracer per unit area and time. The measurements become:

$$
\begin{aligned}
\mu_{i} & =\int_{\Sigma \times T} \sigma_{s}(x, y, t) r_{i}\left(x, y, z_{s}, t\right) d x d y d t \\
& =\int_{\Sigma \times T} \varphi(x, y, t) \sigma_{s}(x, y, t) r_{\varphi i}(x, y, t) d x d y d t
\end{aligned}
$$

This source will be investigated as a combination of the renormalised restrictions $r_{\varphi i}(x, y, t)=\frac{r_{i}\left(x, y, z_{s}, t\right)}{\varphi(x, y, t)}$ with a renormalisation of the domain $\Sigma \times T$.

The interest of the calculations rapidly described in this section is illustrated by a number of figures. The Figs. 2 and 3 describe the stability of the renormalised inversion with respect to an improvement of the resolution. The parts a, c, e, $\mathrm{g}$ of the Figs. 6 and 7 describe in space and time the algebraic and positive estimates. The inversion of synthetic data obtained from the model shows the performance of the inversion because the model is then perfectly representative. The gap between the synthetic and real inversions rather shows the quality of the model and measurements. Nevertheless the understanding of even these figures may not be complete without an account of the theoretical justifications underlying the calculations. This is the task of the following sections.

\section{Calculations about ETEX1}

The experiment ETEX, sponsored by the European Commission, was organised in 1994. A extensive description is given in (Joint Res. Centre, 1998). It was originally aimed, after the Chernobyl event in 1986, at validating the dispersion models that would be involved in forecasting the extension of a polluted plume. A first release ETEX1 was performed near the village of Monterfil, $2^{\circ} 00^{\prime} \mathrm{W}, 48^{\circ} 03^{\prime} \mathrm{N}$, Brittany, France. At ground level $340 \mathrm{~kg}$ of an inert tracer, perfluoromethylcyclohexane or pmch, were emitted on October 1994, between the $23 \mathrm{~d}, 16: 00 \mathrm{UT}$ and the 24 th, 04:00 UT. All over Europe 168 detectors delivered three hourly averaged concentration measurements. These data are available from the Joint Research 


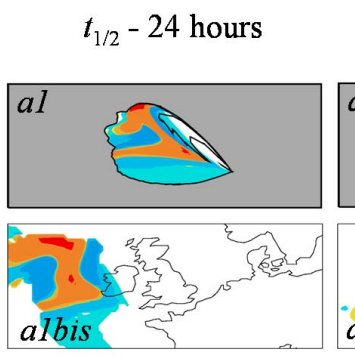

$$
t_{1 / 2}-12 \text { hours }
$$

$t_{1 / 2}: 23 / 10 / 94,22 \mathrm{UT}$

$t_{1 / 2}+12$ hours
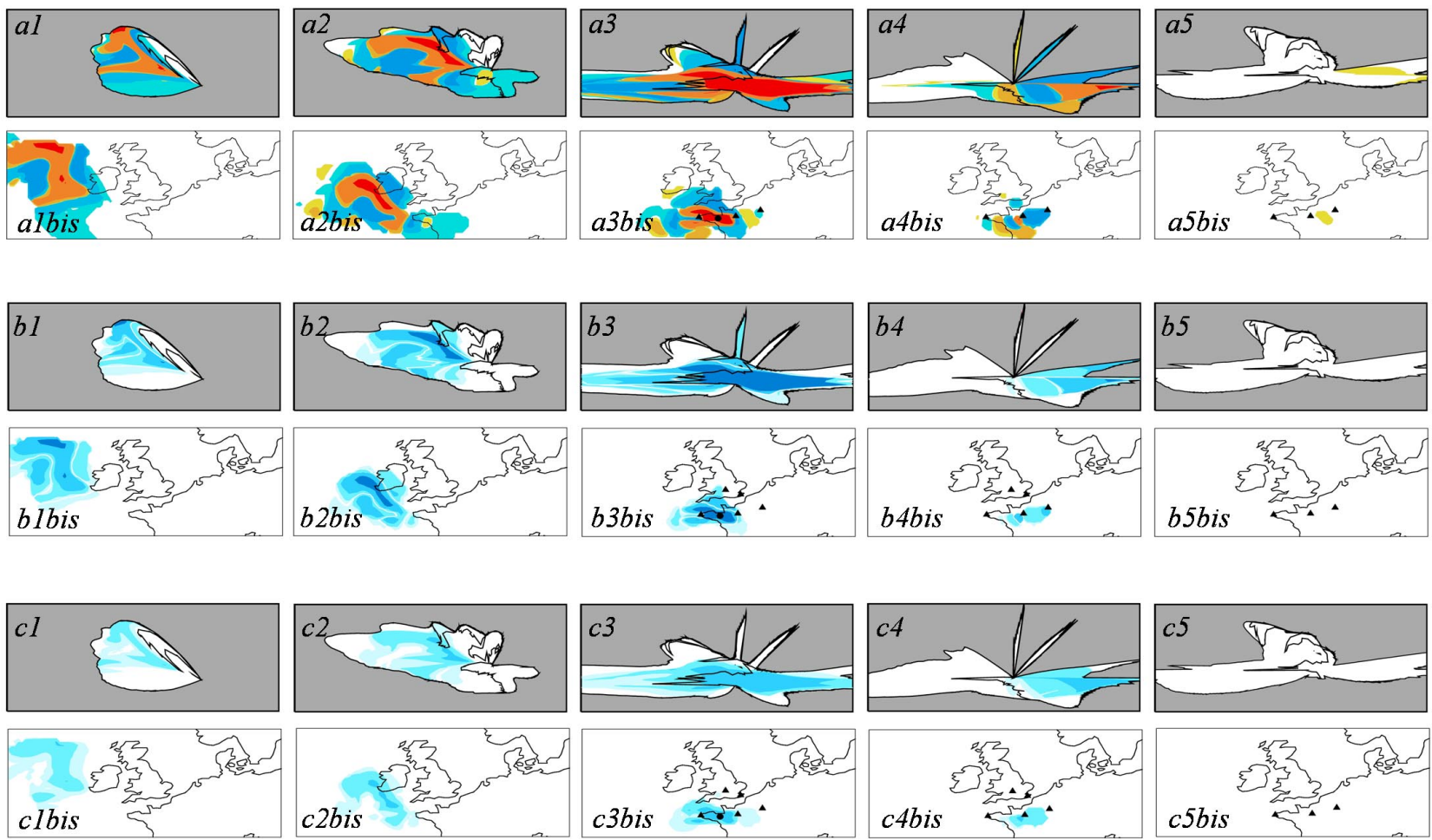

$t_{1 / 2}+24$ hours
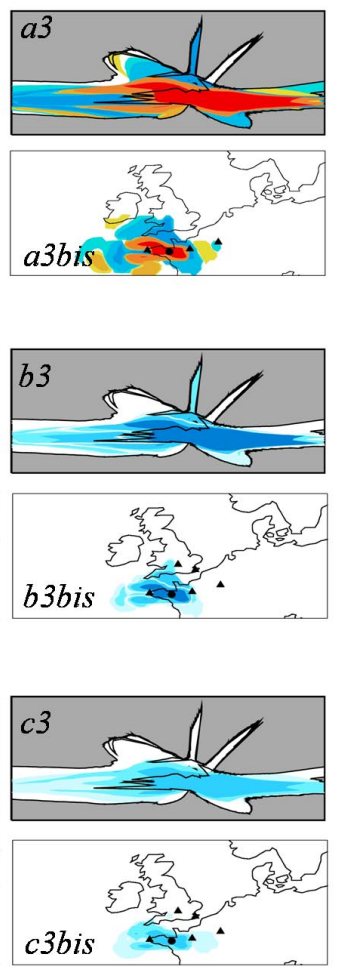

c3bis

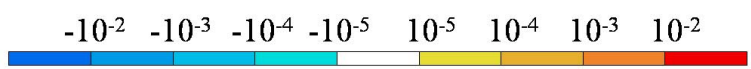

$3.4 \mathrm{~kg} \cdot \mathrm{km}^{-2}$ day $^{-1} \quad(a)$
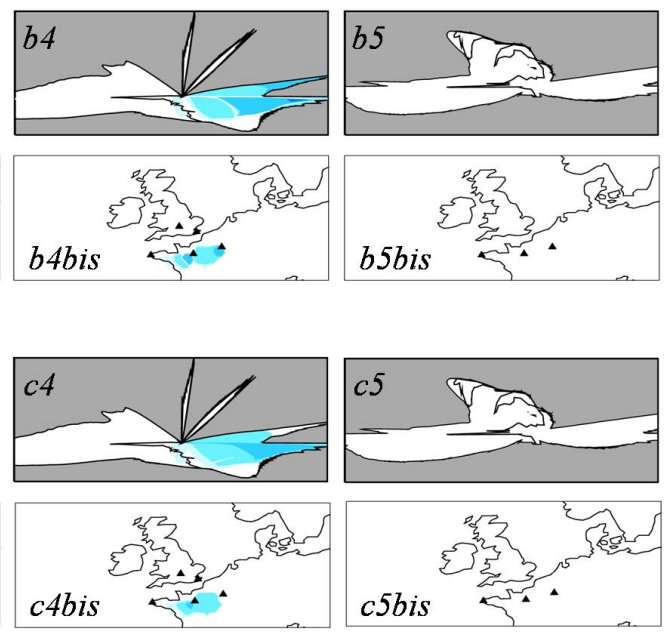

c5bis

$\frac{10^{-9} \quad 10^{-8} \quad 10^{-7} \quad 10^{-6} \quad 10^{-5}}{\left(3.4 \mathrm{~kg} \mathrm{~km}^{-2} \mathrm{day}^{-1}\right)^{2} \quad(b, c)}$

Fig. 8. The source $\sigma$ rebuilt for 51 synthetic ETEX1 measurements is shown in (a) with the renormalised and usual geometries. The space time density of informational energy is described by $\varphi \sigma_{\| \varphi}^{2}$ in the ordinary geometry but in the renormalised geometry of the known domain it becomes $\sigma_{\| \varphi}^{2}$ as shown in (b). The part (c) shows the variance $\overline{\delta \sigma_{\| \varphi}^{2}}$ of this estimate for $30 \%$ of relative Gaussian errors: $\sqrt{\delta \mu_{i}^{2}}=0.3 \mu_{i}$. The quantities $\sigma_{\| \varphi}, \sigma_{\| \varphi}^{2}$ and $\overline{\delta \sigma_{\| \varphi}^{2}}$ are represented 12 hourly at five moments without any time integration; the third moment is the middle of the twelve hour ETEX1 release. The renormalised geometry is accounted for by a radial transformation, centred at Monterfil. As a help, on the figures bis $\sigma_{\| \varphi}, \sigma_{\| \varphi}^{2}$ and $\overline{\delta \sigma_{\| \varphi}^{2}}$ are represented in the ordinary geometry; take care that in this ordinary geometry $\sigma_{\| \varphi}^{2}$ is not a relevant description of the energy. The dot and the triangles indicate the source and the detectors operated at the given moments.

Centre, Environment Monitoring Unit, Ispra (Varese), Italy (web site http://java.ei.jrc.it/etex/database/). The second release ETEX2 performed later in November was considered less successful.

The calculations proposed here, related to ETEX1, were achieved for two selections of 51 measurements performed by 6 detectors and 137 measurements performed on a wider area and longer duration by 14 detectors. The measurements of the first selection are included in the second one except for half of those performed close to Monterfil by the station F2. These selections with the concentrations really observed are presented on the Fig. 1. The stations and measurements were chosen to capture the evolution of the plume of pmch with its edges. In particular, the series of zero measurements taken by the station F8, Brest, should indicate a western limit of the reconstructed release. This limit is not obtained so clearly in the calculations because the series of measurements began at 13:00 UT, just three hours before the release. Data collected earlier would have indicated zero concentrations so that it would be justified to use artificial measurements. We did not do it and the lack of data before 23/10/94, 13:00 UT is expected to harm the quality of the inversion for the early period.

In a first stage the adjoint concentrations $r_{i}$ were calculated in pure advection-diffusion with a time step of $15 \mathrm{~min}$ by the compressible version of the model POLAIR3D. The result was stored hourly at ground level. The calculation was performed on a grid extending from $25^{\circ} \mathrm{W}$ to $30^{\circ} \mathrm{E}$ and from $43^{\circ} \mathrm{N}$ to $68^{\circ} \mathrm{N}$. 


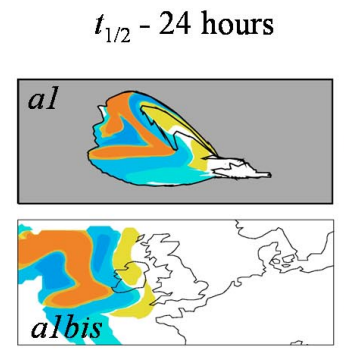

$$
t_{1 / 2}-12 \text { hours }
$$

$t_{1 / 2}: 23 / 10 / 94,22 \mathrm{UT}$

$t_{1 / 2}+12$ hours

$t_{1 / 2}+24$ hours
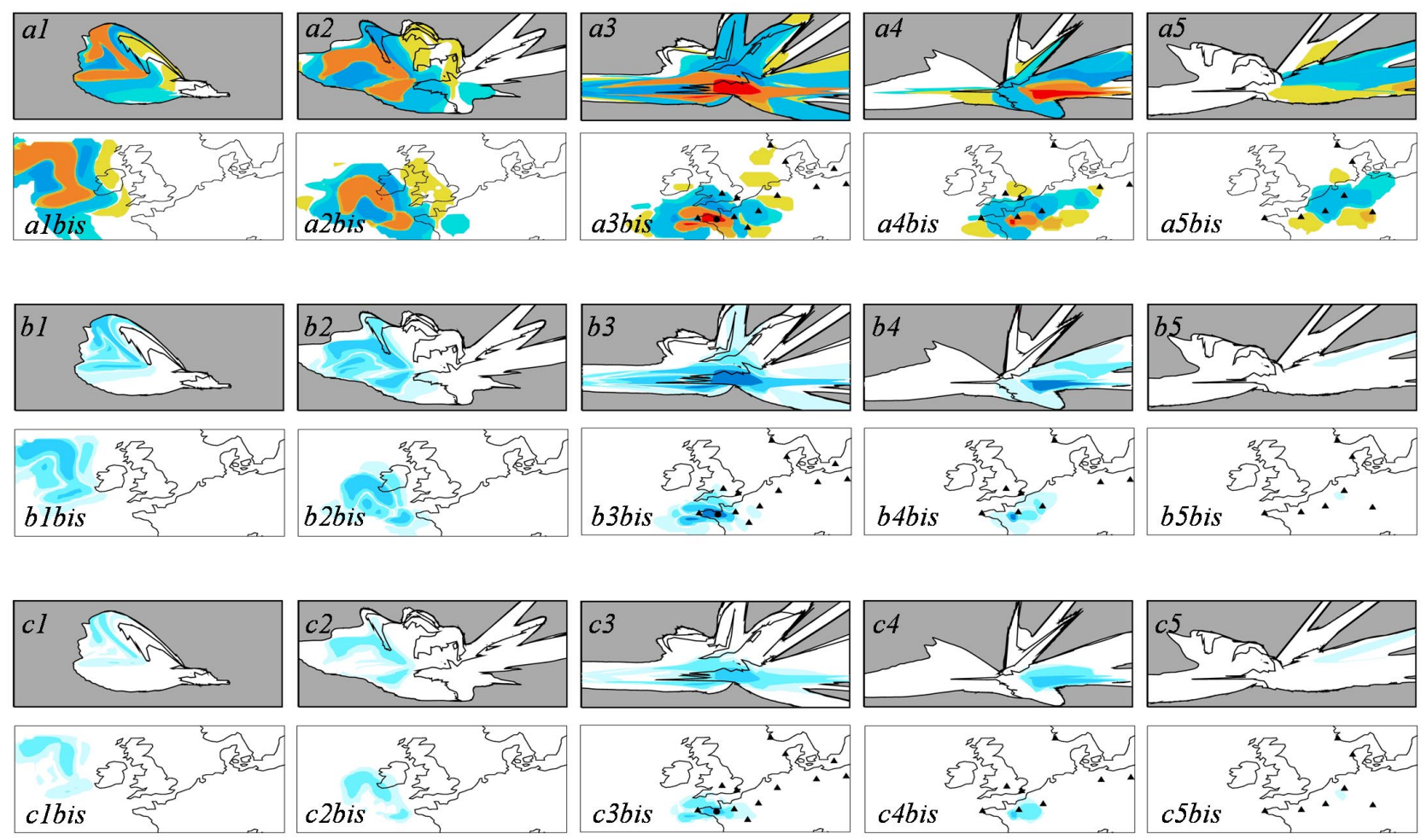

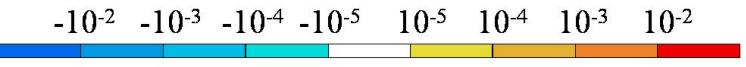

$3.4 \mathrm{~kg} \cdot \mathrm{km}^{-2}$ day $^{-1} \quad(a)$

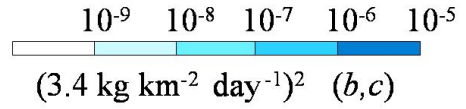

Fig. 9. Results obtained for the selection of 137 measurements. The organisation of this figure is parallel to that of Fig. 8 . One may notice in (b) that, corresponding to the positive and negative parts of the estimate, the informational energy is distributed into two regions separated by a zero line. The zero line could not be seen on the Figs. 6, 7 due to the time integration. The extraction of the level contours from the ordinary geometry was programmed using the marching squares algorithm (Lorensen and Cline, 1987).

In order to illustrate the influence of the resolution on the inversion two sets of adjoint $r_{i}$ were prepared with a horizontal resolution of $0.5^{\circ} \times 0.5^{\circ}$ or $0.25^{\circ} \times 0.25^{\circ}$. The retroplumes are emitted by detectors extending for three hours on an entire mesh; so their size numerically depends on the resolution. Fifteen Cartesian levels were used with boundaries at $0,50,100,200, . ., 2300,2600,3000 \mathrm{~m}$ above ground or sea level. The calculations with time reversal extended from 27/10/94, 07:00 UT back to 19/10/94, 00:00 UT. The sixhourly meteorological data were produced by and obtained from the European Centre for Mediumrange Weather Forecast with the kind agreement of Météo France. The diffusion was parametrised according to Louis (1979) and Louis et al. (1982). POLAIR3D (Sartelet et al., 2002; Sportisse et al., 2002; Boutahar et al., 2003) is the fruit of a close cooperation with the team in charge at Electricite de France of the passive atmospheric transport model Diffeul (Wendum, 1998). It is a fully modular 3D Eulerian chemistry transport model. Advection is solved with a flux limiter method; diffu- sion is solved by a three point scheme. A general account of the numerical treatment of the diffusion was given by Ouahsine and Smaoui (1999). Each adjoint concentration required $2 \mathrm{mn}$ CPU time for the coarse resolution, $8 \mathrm{mn}$ for the thin resolution, on a PC (Pentium IV, $2.8 \mathrm{GHz}, 1 \mathrm{Go}$ RAM).

In a second stage the inversion was performed according to the method developed in this paper for a surface source at ground or sea level. The period of time under investigation extended from 19/10/94, 00:00 UT until 27/10/94, 07:00 UT with a step of one hour. The area extended between $24.5^{\circ} \mathrm{W}$ to $23.5^{\circ} \mathrm{E}$ and from $43.5^{\circ} \mathrm{N}$ to $67.5^{\circ} \mathrm{N}$ and both horizontal resolutions were used, $0.5^{\circ} \times 0.5^{\circ}$ or $0.25^{\circ} \times 0.25^{\circ}$. Note that the coarse and thin inversions were performed with the same sets of synthetic measurements produced by the coarse model. The comparison is accordingly freed from the direct effect of the resolution on the measurements. Four sets of data were used: 
1. synthetic values $\boldsymbol{\mu}^{s}$ calculated with the resolution of $0.5^{\circ} \times 0.5^{\circ}$ by POLAIR3D for both selections of measurements and for the source ETEX1

2. real values $\boldsymbol{\mu}$ from the ETEX1 database for both selections

3. synthetic values $\boldsymbol{\mu}^{g}$ calculated in resolution $0.5^{\circ} \times 0.5^{\circ}$ for the selection of 137 measurements and for an imaginary widespread Gaussian source

4. synthetic values $\boldsymbol{\mu}^{p}$ of 137 measurements in resolution $0.5^{\circ} \times 0.5^{\circ}$ for a point source of $340 \mathrm{~kg}$ close to or inside the mesh of the station F2.

The reconstruction of a Gaussian source was prepared in order to investigate the potential of the method with respect to the widespread sources for which it is primarily designed. The Gaussian source was placed in a space time region of good illumination with a centre at $8^{\circ} \mathrm{E}, 49^{\circ} \mathrm{N}$ in space, on 24/10/94, 10:00 UT in time, six hours after the end of the ETEX1 release. The total amount of the release was again $340 \mathrm{~kg}$ for characteristic length and duration $l_{0}=165 \mathrm{~km}$, $\tau=18$ hours. This imaginary release was accordingly proportional to the Gaussian distribution $\gamma(l, t)$ for a distance $l$ and a date $t$ measured from the centre:

$\gamma(l, t)=\frac{e^{-\frac{1}{2}\left\{\frac{l^{2}}{l_{0}^{2}}+\frac{t^{2}}{\tau^{2}}\right\}}}{(2 \pi)^{\frac{3}{2}} \tau^{\frac{1}{2}} l_{0}}$

The reconstructions are complemented by a series of diagnostics described in the forthcoming sections: the use of a renormalised geometry (Sects. 5, 6, 10), the response of the estimate to measurement errors (Sect. 8), the informational energy (Sect. 9). The images in the renormalised geometry were prepared in vector format. The vectorial images are defined with an accuracy independent of the visualisation tools by contours, and the contours are defined as polygons by a list of points. In our case the contours were tied to the coast lines and to function levels. The emission, energy and variance levels had to be extracted from the ordinary geometry as indicated in the comments of the Fig. 9. Each point of the contours was then moved according to the Eq. (46). To this end the function $\varphi$ calculated with a space grid $96 \times 48$ was interpolated on a much thiner grid $672 \times 336$.

The calculation time for the coarse inversion with 51 measurements was $2 \mathrm{mn} 30 \mathrm{~s}$ CPU on a PC (Pentium IV, $2.8 \mathrm{GHz}, 1 \mathrm{Go}$ RAM). With 137 measurements this time becomes $20 \mathrm{~min}$. These delays are roughly multiplied by 4 for the thin inversions. Most of this time is devoted to the iterative calculation of the renormalising function. The calculation of the algebraic and positive estimates was organised in order to require then a few additional seconds. The transfer of the results into the renormalised geometry required a few seconds of computational time.

\section{Geometry and statistics}

This section is a preliminary comparison of the function $f$ introduced in this work through geometric considerations and the background error covariance matrix defined in usual assimilation based on statistical arguments. We shall see that the comparison meets a geometric obstacle and a statistical one, both to be further investigated in the next section before addressing the optimal choice of the renormalising function in the Sec. 7.

The following geometric discussion is the starting point of the comparison and will be later the basis of the transformation, illustrated on the Fig. 4, of the illumination into a curved geometry. The usual Eq. (18) of data assimilation is traditionally presented in a discretised form with a finite number $v_{\max }$ of meshes at mean space-time positions $\boldsymbol{x}^{\nu}$ of space-time weights $d^{v}$ (corresponding to the integral element $\rho d \boldsymbol{x})$. We keep the same notation $\sigma$ for the continuous source $\sigma(\boldsymbol{x})$ and the corresponding column vector integrated in each mesh with elements: $\sigma_{\nu}=d^{v} \sigma\left(\boldsymbol{x}^{\nu}\right)$. Most often $\sigma$ is rather intended as the mismatch between the sought source and an a priori estimate; $\boldsymbol{\mu}$ is then the mismatch between the measurements really observed and the values synthesised for $\sigma_{\text {pri }}$ by the available model. The inversion of $\mu_{1}, \ldots, \mu_{n}$ is usually addressed with a functional $\mathcal{J}$. The estimate $\sigma^{\text {est }}$ of the unknown real source $\sigma$ is the source $s$ minimising $\mathcal{J}(s)$ :

$$
\begin{aligned}
& \mathcal{J}(s)={ }^{t} s \mathbf{B}^{-1} s+{ }^{t}(\mathbf{R} s-\boldsymbol{\mu}) \mathbf{Q}^{-1}(\mathbf{R} s-\boldsymbol{\mu}) \\
& \left\langle s, s^{\prime}\right\rangle_{b}={ }^{t} s \mathbf{B}^{-1} s^{\prime}
\end{aligned}
$$

The $v_{\max } \times v_{\max }$ covariance matrix of the estimation error or background error matrix is $\mathbf{B}=\overline{\sigma^{t} \sigma}$, the bar standing for the statistical expectation. The related part of the cost function defines a scalar product $\langle,\rangle_{b}$. The $n \times v_{\max }$ matrix $\mathbf{R}$ stands for the observation operator, or rather, its linear or linearised deterministic part; the lines of $\mathbf{R}$ correspond to our $r_{i}$. The measurements $\boldsymbol{\mu}=\mathbf{R} \sigma+\delta \boldsymbol{\mu}$ include a noise described by the $n \times n$ covariance matrix $\mathbf{Q}=\overline{\delta \boldsymbol{\mu} t} \delta \boldsymbol{\mu}$. This noise is due on the one hand to the technical performance of the detectors, on the other hand to the quality of the model from which $\mathbf{R}$ is evaluated. So far the transport model and measurements were considered perfect which amounts to taking an infinite $\mathbf{Q}^{-1}$ and to minimising ${ }^{t} s \mathbf{B}^{-1} s$ under the constraints that $s$ strictly obeys the measurements, $\mathbf{R} s=\boldsymbol{\mu}$.

In the present context there is no explicit a priori estimate. It is possible to consider that the null source plays this role. Accordingly, $\mathbf{B}=\overline{\sigma^{t} \sigma}$ might be seen as the covariance matrix of the source investigated by the measurements.

The continuous descriptions of $\mathbf{B}$ and $\mathbf{B}^{-1}$ are through inverse kernels $b(\boldsymbol{x}, \boldsymbol{y})=\overline{\sigma(\boldsymbol{x}) \sigma(\boldsymbol{y})}$ and $b^{-}$: $\left\langle\sigma, \sigma^{\prime}\right\rangle_{b}=\int \sigma(\boldsymbol{x}) b^{-}(\boldsymbol{x}, \boldsymbol{y}) \sigma^{\prime}(\boldsymbol{y}) d \boldsymbol{x} d \boldsymbol{y}$. The diagonal elements of the discretised matrix $\mathbf{B}$ are, by definition, $b_{v v}=\overline{\sigma_{v}^{2}}=\left(d^{v} \sigma\left(\boldsymbol{x}^{v}\right)\right)^{2}$. In the geometry of $\mathbf{B}^{-1}$ a vector source is transformed as $\tilde{\sigma}=\sqrt{\mathbf{B}^{-1}} \sigma$ (Loren, 1988) in such a way that ${ }^{t} \sigma \mathbf{B}^{-1} \sigma^{\prime}$ becomes ${ }^{t} \tilde{\sigma} \tilde{\sigma}^{\prime}$ with the identity 
as new background covariance matrix. If $\mathbf{B}$ was diagonal so would be $\mathbf{B}^{-1}$ with elements $\frac{1}{b_{v v}}$. A source would become $\tilde{\sigma}_{\nu}=\frac{d^{v} \sigma\left(\boldsymbol{x}^{\nu}\right)}{b_{\nu \nu}}$ and, in compensation of the simplified background matrix, the weights $d^{v}$ of the meshes would be replaced by $\frac{d^{\nu}}{\sqrt{b_{\nu v}}}$. This diagonal example shows that $\mathbf{B}^{-1}$ transforms the subspace $\Omega \times \mathrm{T}$ of $\mathbb{R}^{4}$ into a curved space in which, as $\overline{\tilde{\sigma}_{v}^{2}}=1$, the information is equally distributed. Hence we shall say that $\Omega \times \mathrm{T}$ endowed with the product $\langle,\rangle_{b}$ is a version of the known domain. The non vanishing off diagonal terms $b_{v v^{\prime}}$ of $\mathbf{B}^{-1}$ indicate a degree of confusion between the meshes $v$ and $v^{\prime}$ that cannot be totally distinguished. When $\mathbf{B}^{-1}$ or the kernel $b^{-}$are purely diagonal the known domain will be called "separate".

The strategy of the previous section amounts to minimising a norm $(s, s)_{f}=\int \rho f s^{2} d \boldsymbol{x}$ under the constraints that $s$ strictly meets the measurements $\mu_{i}$. The choice of $f$ may then be suspected to be somehow a hypothesis about the form of $\mathbf{B}$ (in fact $\mathbf{B}^{-1}$ ) and $\langle,\rangle_{b}$. The renormalising function may be represented by a diagonal matrix:

$\mathbf{F}=\left[\begin{array}{lll}f^{1} & \\ & \cdot & \\ & & f^{v_{\max }}\end{array}\right]$

The straightforward link $\mathbf{B}=\mathbf{F}^{-1}$ or $\langle,\rangle_{b}=(,)_{f}$ is all the more tempting as both objects are aimed at describing which regions are well or badly monitored. There come the announced two obstacles.

The first obstacle is geometric. If $\mathbf{B}$ was diagonal the identification $f^{v}=\frac{1}{b_{v v}}$ could be reasonable. By definition $\mathbf{F}$ is diagonal but $\mathbf{B}^{-1}$ in general is not. This would imply a separate geometry of the observed domain with vanishing correlations $\overline{\sigma(\boldsymbol{x}) \sigma(\boldsymbol{y})}=0$ unless $\boldsymbol{x}=\boldsymbol{y}$.

The second obstacle is statistical. The definition of $\mathbf{B}$ with the kernel $b(\boldsymbol{x}, \boldsymbol{y})=\overline{\sigma(\boldsymbol{x}) \sigma(\boldsymbol{y})}$ explicitly refers to some probability distribution of the source. These statistics are classically tied to the quality of some a priori model for the source (besides the transport model). No source model enters the definition of $\mathbf{F}$. In the classical theory this a priori model is generally not carefully provided because $\mathbf{B}$ can be iteratively clarified by an a posteriori use of the measurements successively available. The Kalman-Bussy like procedure (Rödenbeck et al., 2003; Anderson and Moore, 1979) is based on the statistics of the measurement errors due to the transport model and detectors. But the context of $\mathbf{F}$ is error-free: the transport model and detectors might as well be a perfect reality.

In the next section both obstacles are explored. The geometric one will be arranged by showing that $(,)_{f}$ is physically equivalent to a non separate product $\langle,\rangle_{f}$ more reasonably identifiable to $\langle,\rangle_{b}$. Because of the statistical obstacle a link between $\mathbf{B}$ and $\mathbf{F}$ cannot be addressed as a mathematical requirement, but rather as a physically motivated assumption implying a hypothesis about the probability distribution of the source investigated by the measurements. We shall nevertheless obtain statistics of nature completely different from the classical considerations about technical errors.

\section{An assumption}

The statistical obstacle above suggests that a probability distribution might be assigned to the source regardless of any a priori model. Let's use a dotted bar to denote such hypothetical statistics based on which a covariance matrix or kernel $\dddot{\sigma}(\boldsymbol{x}) \sigma(\boldsymbol{y})$ might be defined. A source $\sigma=\sigma_{\| f}+\sigma_{\perp f}$ may always be decomposed into its parts parallel and orthogonal to the $r_{f i}$ :

$$
\begin{aligned}
& \widetilde{\sigma(\boldsymbol{x}) \sigma(\boldsymbol{y})}=\widetilde{\sigma}_{\| f}(\boldsymbol{x}) \sigma_{\| f}(\boldsymbol{y}) \\
& +\widetilde{\sigma}_{\| f}(\boldsymbol{x}) \sigma_{\perp f}(\boldsymbol{y})+\widetilde{\sigma}_{\perp f}(\boldsymbol{x}) \sigma_{\| f}(\boldsymbol{y}) \\
& +\sigma_{\perp f}(\boldsymbol{x}) \sigma_{\perp f}(\boldsymbol{y})
\end{aligned}
$$

with:

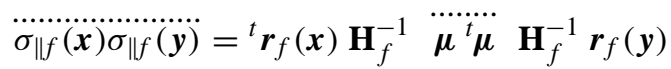

The last expression contains the measurement (or mismatch) covariance matrix $\widetilde{\boldsymbol{\mu}}^{t} \boldsymbol{\mu}$ not to be mistaken for a measurement error covariance matrix $\mathbf{Q}=\overline{\delta \boldsymbol{\mu}^{t} \delta \boldsymbol{\mu}}$. If the information available from the measurements is optimally used in restoring $\sigma_{\| f}$ then $\sigma_{\perp f}$ is essentially unknown and undetermined. The terms in the second line of the Eq. (20) should vanish and also the term in the third line when $\boldsymbol{x} \neq \boldsymbol{y}$. The reasonable assumption that $\widetilde{\sigma(\boldsymbol{x}) \sigma(\boldsymbol{y})}$ is a continuous function, combined with the continuity of the Eq. (21), implies that $\sigma_{\perp f}(\boldsymbol{x})^{2}$ should vanish too. We think this inconsistency just means that $\sigma_{\perp f}$ is not a relevant physical entity; about it nothing is known, nothing can be said even statistically. Note that, from a purely mathematical point of view, if the subspace of the sources parallel to the $r_{f i}$ is well defined, that of the perpendicular sources cannot be defined in a unique unambiguous way. A more physical point of view may be obtained by decomposing $(,)_{f}$ as:

$(\sigma, \sigma)_{f}=\left(\sigma_{\| f}, \sigma_{\| f}\right)_{f}+\left(\sigma_{\perp f}, \sigma_{\perp f}\right)_{f}$

The only term in this expression that may be calculated from the observations is $\left(\sigma_{\| f}, \sigma_{\| f}\right)_{f}$. The term $\left(\sigma_{\perp f}, \sigma_{\perp f}\right)_{f}$ is not knowable so that its physical status is indeed questionable. This discussion implies that if a statistics is to be introduced to describe the sources likely to be observed, it depends on the choice of $f$. Accordingly we denote appropriately labelled the covariance matrix and kernel as:

$$
\begin{aligned}
& \mathbf{B}_{f}, \quad b_{f}(\boldsymbol{x}, \boldsymbol{y})=\widetilde{\sigma}(\boldsymbol{x}) \sigma(\boldsymbol{y})
\end{aligned}
$$

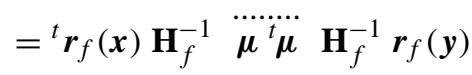

Several points must be stressed. Firstly the statistics gives no indication about the difference $\sigma-\sigma_{\| f}=\sigma_{\perp f}$ which cannot 
be attributed here any physical relevance as a reconstruction error. The statistics is relevant only for the $n$-dimensional vector space containing the observable and rebuildable part $\sigma_{\| f}$. Secondly, the statistics depends on $f$ which seems to interfere now with the definition of the physical reality. This is an indication that $f$ should not be chosen arbitrarily as discussed is the next section. Thirdly, the choice of $f$ is not completely constraining for the hypothetical statistics: $\boldsymbol{\mu}^{t} \boldsymbol{\mu}$ is still free. It is nevertheless clear from the Eq. (23) that $\mathbf{B}_{f}$ is not diagonal, that the scalar product $\langle,\rangle_{b f}$ hereafter defined parallel to the definition 18 is not separate as $(,)_{f}$ is. Let's denote:

$$
\left\langle\sigma, \sigma^{\prime}\right\rangle_{b f}={ }^{t} \sigma \mathbf{B}_{f}^{-1} \sigma^{\prime} \quad\left\langle\sigma, \sigma^{\prime}\right\rangle_{f}=\left(\sigma_{\| f}, \sigma_{\| f}^{\prime}\right)_{f}
$$

The second above definition still provides a scalar product of $\sigma$ because $\sigma_{\| f}=\mathbf{P}_{f} \sigma$ is a linear function. Denoted in matrix form, $\mathbf{P}_{f}$ is an orthogonal projector, for $(,)_{f}$, on the observable space spanned by the $r_{f i}$ : it is self adjoint and $\mathbf{P}_{f}^{2}=\mathbf{P}_{f}$. The kernel $p_{f}$ may be obtained by putting the form 8 of $\boldsymbol{\mu}$ into the expression 9 of $\sigma_{\| f}$ :

$$
\begin{aligned}
& \sigma_{\| f}(\boldsymbol{x})=\int_{\Omega \times T} \sigma(\boldsymbol{y}) f(\boldsymbol{y}){ }^{t} \boldsymbol{r}_{f}(\boldsymbol{y}) \mathbf{H}_{f}^{-1} \boldsymbol{r}_{f}(\boldsymbol{x}) \rho(\boldsymbol{y}) d \boldsymbol{y} \\
& p_{f}(\boldsymbol{x}, \boldsymbol{y})=f(\boldsymbol{y}){ }^{t} \boldsymbol{r}_{f}(\boldsymbol{y}) \mathbf{H}_{f}^{-1} \boldsymbol{r}_{f}(\boldsymbol{x})
\end{aligned}
$$

Despite their mathematical difference, $(,)_{f}$ and $\langle,\rangle_{f}$ are physically equivalent with restrictions explained in the Sec. 9. In particular the $r_{f i}$ are adjoint to the measurements for both $(,)_{f}$ and $\langle,\rangle_{f}$ :

$\mu_{i}=\left(\sigma, r_{f i}\right)_{f}=\left\langle\sigma, r_{f i}\right\rangle_{f}$

The matrix associated to $(,)_{f}$ is $\mathbf{F}$, that tied to $\langle,\rangle_{f}$ is $\mathbf{P}_{f} \mathbf{F} \mathbf{P}_{f}=\mathbf{P}_{f} \mathbf{F}=\mathbf{F} \mathbf{P}_{f}$. This is no longer a full rank diagonal matrix so that $\langle,\rangle_{f}$ is not a separate product. The following identification becomes conceivable:

$$
\langle,\rangle_{f}=\langle,\rangle_{b f} \quad \text { or } \quad \mathbf{B}_{f}^{-1}=\mathbf{P}_{f} \mathbf{F}=\mathbf{F P}_{f}
$$

As already stressed by the Sect. 5, this decision of the ambiguous link between $\mathbf{B}_{f}$ and $\mathbf{F}$ cannot be demonstrated, it must be admitted as a definition or as an assumption. The arguments developed so far made it reasonable. It is made even more consistent with the intuition by the resulting Eq. (29).

According to the assumption 27 the rank of $\mathbf{B}_{f}^{-1}$, bounded by the number of measurements, is not full. The inverse notation might be questioned but there will be no problem as long as the very working space is that spanned by the $r_{f i}$. As $\mathbf{B}_{f}^{-1}=\mathbf{P}_{f} \mathbf{F}$ or $\mathbf{B}_{f}=\mathbf{F}^{-1} \mathbf{P}_{f}$ with a diagonal $\mathbf{F}$ we get from the Eq. (25):

$b_{f}(\boldsymbol{x}, \boldsymbol{y})={ }^{t} \boldsymbol{r}_{f}(\boldsymbol{y}) \mathbf{H}_{f}^{-1} \boldsymbol{r}_{f}(\boldsymbol{x})$

Comparing with the Eq. (23) we find that the assumption 27 leads to expect measurement values according to a law with a covariance matrix:

$$
\boldsymbol{\mu}^{t} \ddot{\boldsymbol{\mu}}=\mathbf{H}_{f} \quad \text { or } \quad \dddot{\mu}_{i} \dddot{\mu}_{j}=\left(r_{f i}, r_{f j}\right)_{f}=\left\langle r_{f i}, r_{f j}\right\rangle_{f}
$$

In particular if two detectors have adjoint functions $r_{f i}, r_{f j}$ that do not overlap, the correlation between the expectable measurements vanishes: $\dddot{\mu}_{i} \dddot{\mu}_{j}=0$. For the continuation the Eq. (27) will be complemented by the assumption that the measurements are expected according to a Gaussian law with

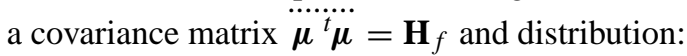

$q_{f}(\boldsymbol{\mu})=\frac{e^{-\frac{1}{2}\left\{{ }^{t} \boldsymbol{\mu} \mathbf{H}_{f}^{-1} \boldsymbol{\mu}\right\}}}{\sqrt{(2 \pi)^{n} \operatorname{det} \mathbf{H}_{f}}}$

The geometric obstacle of the Sect. 5 that $\mathbf{F}$ is diagonal has been overcome. The assumption 27 did not totally remove the statistical obstacle because, contrary to the classical theory, the statistics assigned to each function $f$ are independent of any a priori source model; $\mathbf{B}_{f}$ above does not fit with the classical interpretation of a background error covariance matrix B. The above statistics do not rely on technical errors. They correspond to the following question before the measurements are effectively known: which sources $\sigma$ and measurements $\boldsymbol{\mu}$ we may expect to observe? The question is independent of the technical quality of the detectors. This makes sense: $\sigma$ is supposed to exist independently from its observation and from the quality of its observation. Once $f$ will have been optimised in the Sect. 7, we will propose to designate these statistics with the word "anticipations".

\section{Intrinsically relevant information}

The function $f$ was introduced based on the idea that weights could be attributed to the various parts of $\Omega \times \mathrm{T}$ in order to interpret the measurements. In the Sects. 5 and 6 we realised these weights also had a statistical meaning interfering with the definition of a physical reality. This conclusion could be perplexing but it happens that a unique function consistently achieves the idea of a weight distribution. This unique optimal renormalising function, denoted $\varphi(\boldsymbol{x})$, will be seen furthermore to minimise the information retrieved from the observations thus avoiding such inversion artefacts as those visible about the detectors on the parts a, $\mathrm{c}$ of the Figs. 2 and 3. The calculation of $\varphi$ depends of the space time position of the detectors and meteorological conditions. Shortly, it is a function of the observation setup regardless of its technical quality (the measurement errors), also regardless of the values effectively observed.

The renormalising function should be chosen among functions of a given total weight, for instance and for the sake of further clarity, $n$, the number of pieces of information. So $f$ will be termed acceptable provided that:

$\boldsymbol{r}(\boldsymbol{x}) \neq 0 \Longrightarrow f(\boldsymbol{x})>0$ and $\int_{\Omega \times \mathrm{T}} \rho f d \boldsymbol{x}=n$

The Eq. 10 shows the modified illumination $E_{f}(x)$ to be another acceptable weight. The weight $E_{f}(\boldsymbol{x})$ is attributed to $\boldsymbol{x}$ by the inversion based on the previous choice of a weight 
$f(\boldsymbol{x})$. Hence the weighting strategy becomes inconsistent unless $E_{f}=f$. Thus $\varphi$ should be chosen in such a way that:

$\boldsymbol{r}(\boldsymbol{x}) \neq 0 \Longrightarrow{ }^{t} \boldsymbol{r}_{\varphi}(\boldsymbol{x}) \mathbf{H}_{\varphi}^{-1} \boldsymbol{r}_{\varphi}(\boldsymbol{x})=1$ i.e. $E_{\varphi}=\varphi$

This equation selects a unique optimal $\varphi$ because, as shown in Appendix A, it is the minimality condition for $\operatorname{det} \mathbf{H}_{f}$ which is positive and convex among all acceptable functions. We may now unambiguously define as anticipations the dotted statistics from the Sect. 6 tied to the present choice of $\varphi$.

The minimality of $\operatorname{det} \mathbf{H}_{\varphi}$ may be obtained as a more physical entropic requirement. Roughly speaking, the amount of information obtained from the measurements depends on the choice of $f$ and, in order to avoid inversion artefacts, this amount should be minimised.

The entropy of an experiment with $N$ discrete possibilities having probabilities $q_{i}$ was formulated by Hartley (1928); Shannon (1948) after the work of Boltzmann in statistical thermodynamics:

$S=-\sum_{i=1}^{N} q_{i} \log q_{i}$

The quantity $-\log q_{i}$ measures the information got by an observer discovering the result number $i$; it may be thought of as the surprise of the observer, weak for very probable events with $q_{i} \simeq 1$, and strong for improbable events. The entropy of information 33 is the expectation of this information, it measures the relevance of the experiment.

If the outcome of the experiment is a vector $\mu$ with a continuous probability distribution function $q(\boldsymbol{\mu})$ the entropy of information generalises as:

$S=-\int q(\boldsymbol{\mu}) \log q(\boldsymbol{\mu}) d \boldsymbol{\mu}$

This generalisation is not as straightforward as it looks like. An account of the problems may be found in (Hatori, 1958; Middleton, 1960). The entropy of the Gaussian distribution 30 for $\mu_{1}, . ., \mu_{n}$ with a covariance matrix $\mathbf{H}_{f}$ is:

$S_{f}=\frac{n}{2} \log (2 \pi)+\frac{1}{2} \log \operatorname{det} \mathbf{H}_{f}$

Thus, as $\operatorname{det} \mathbf{H}_{\varphi}$ is minimised by the condition 32 , the average information expected from the measurements is minimal for $\varphi$. We think the excess of entropy $S_{f}-S_{\varphi} \geq 0$ tied to another geometric choice would correspond to an artificial information and would lead to inversion artefacts. For instance the entropy $S_{1}$ tied to the standard geometry, $f \equiv 1$, is indefinitely large: the diagonal terms $h_{i, i}$ of the covariance matrix $\mathbf{H}$ diverge thus outgrowing the off-diagonal ones. The measurements, attributed artificially to the close environment of the detectors, become pairwise independent. This explains why on the Figs. 10d and 11d, when a higher numerical resolution shrinks the modelled detectors, the negative parts of the non renormalised estimate vanish. The excess of entropy with respect to the intrinsic minimum $S_{\varphi}$ measures our excessive surprise at discovering the estimates of the Figs. 2, 3 parts a, c. When the geometry is optimised the potential sources away from the detectors are duly taken into account.

Let's consider now two situations. In the first situation $n$ measurements $\mu_{1}, \ldots, \mu_{n}$ are performed. In the second situation, exactly the same measurements are performed plus one, $\mu_{n+1}$. Note that the subscript $n+1$ is not a chronological indication that this additional measurement is done after the other ones, it is just one more measurement. The first situation will correspond to an optimal renormalising function $\varphi_{n}$ and the statistics of the measurements will follow a $n \times n$ covariance matrix $\mathbf{H}_{n}$. In the second case an optimal renormalising function $\varphi_{n+1}$ will be obtained with a $(n+1) \times(n+1)$ covariance matrix $\mathbf{H}_{n+1}$ :

$$
\begin{aligned}
& \mathbf{H}_{n}=\left[h_{n, i, j}\right] \quad i=1, \ldots, n \quad j=1, \ldots, n \\
& h_{n, i, j}=\dddot{\mu}_{i} \dddot{\mu}_{j}{ }^{\prime n}=\int_{\Omega \times \mathrm{T}} \rho \frac{r_{i} r_{j}}{\varphi_{n}} d \boldsymbol{x} \\
& \mathbf{H}_{n+1}=\left[h_{n+1, i, j}\right] \quad i=1, \ldots, n+1 \quad j=1, \ldots, n+1 \\
& h_{n+1, i, j}=\dddot{\mu}_{i} \dddot{\mu}_{j}^{/ n+1}=\int_{\Omega \times \mathrm{T}} \rho \frac{r_{i} r_{j}}{\varphi_{n+1}} d \boldsymbol{x}
\end{aligned}
$$

The dotted bars for the statistical anticipations now bear an indication of the situation under examination. There is no reason why $\varphi_{n}$ and $\varphi_{n+1}$ should be equal. Thus $\mathbf{H}_{n}$ does not coincide with the $n \times n$ upper diagonal submatrix $\mathbf{H}_{n+1 / n}$ of $\mathbf{H}_{n+1}$. The mere existence of $\mu_{n+1}$ changes the anticipated statistics of $\mu_{1}, \ldots, \mu_{n}$. This is a at first sight a strange result. We could indeed imagine that we perform the additional measurement but do not look at the result. This is of course an effect of how the additional $r_{n+1}(\boldsymbol{x})$ will overlap with the other $r_{i}(\boldsymbol{x})$ thus altering the system of weights distributed in $\Omega \times \mathrm{T}$.

Note that if $r_{n+1}(\boldsymbol{x})$ did not overlap at all with any of the $r_{i}(\boldsymbol{x}), i \leq n$, then the optimisation of $\varphi_{n+1}$ would split into two separate optimal problems for the domains of $r_{n+1}(\boldsymbol{x})$ on the one hand, of the other $r_{i}(\boldsymbol{x})$ on the other hand. And then the identity $\mathbf{H}_{n}=\mathbf{H}_{n+1 / n}$ would stand. In the reality, two retroplumes always overlap in an early enough past.

Surprisingly the optimal function $\varphi$ is numerically obtained by the simple and straightforward algorithm 13. This algorithm might be read : $f_{k+1}(\boldsymbol{x})=\frac{E_{f_{k}}(\boldsymbol{x})}{\sqrt{{ }_{\boldsymbol{r}_{f_{k}}(\boldsymbol{x}) \mathbf{H}_{f_{k}}^{-1} \boldsymbol{r}_{f_{k}}(\boldsymbol{x})}}}$ thus combining the properties 32 and 10 of the limit $\varphi=E_{\varphi}$ and $\varphi(\boldsymbol{x})=\frac{E_{\varphi}(\boldsymbol{x})}{{ }_{{ }_{\boldsymbol{r}}(\boldsymbol{x}) \mathbf{H}_{\varphi}^{-1} \boldsymbol{r}_{\varphi}(\boldsymbol{x})}}$. It enables to obtain $\varphi$ with any required accuracy and rapidly on any subdomain avoiding the condition $\boldsymbol{r}(\boldsymbol{x})=0$. Its efficiency was always verified though no formal proof was found yet.

\section{Anticipations and measurement errors}

The best renormalising function $\varphi$ generates the dotted anticipated statistics about the future measurements $\boldsymbol{\mu}$ that will 


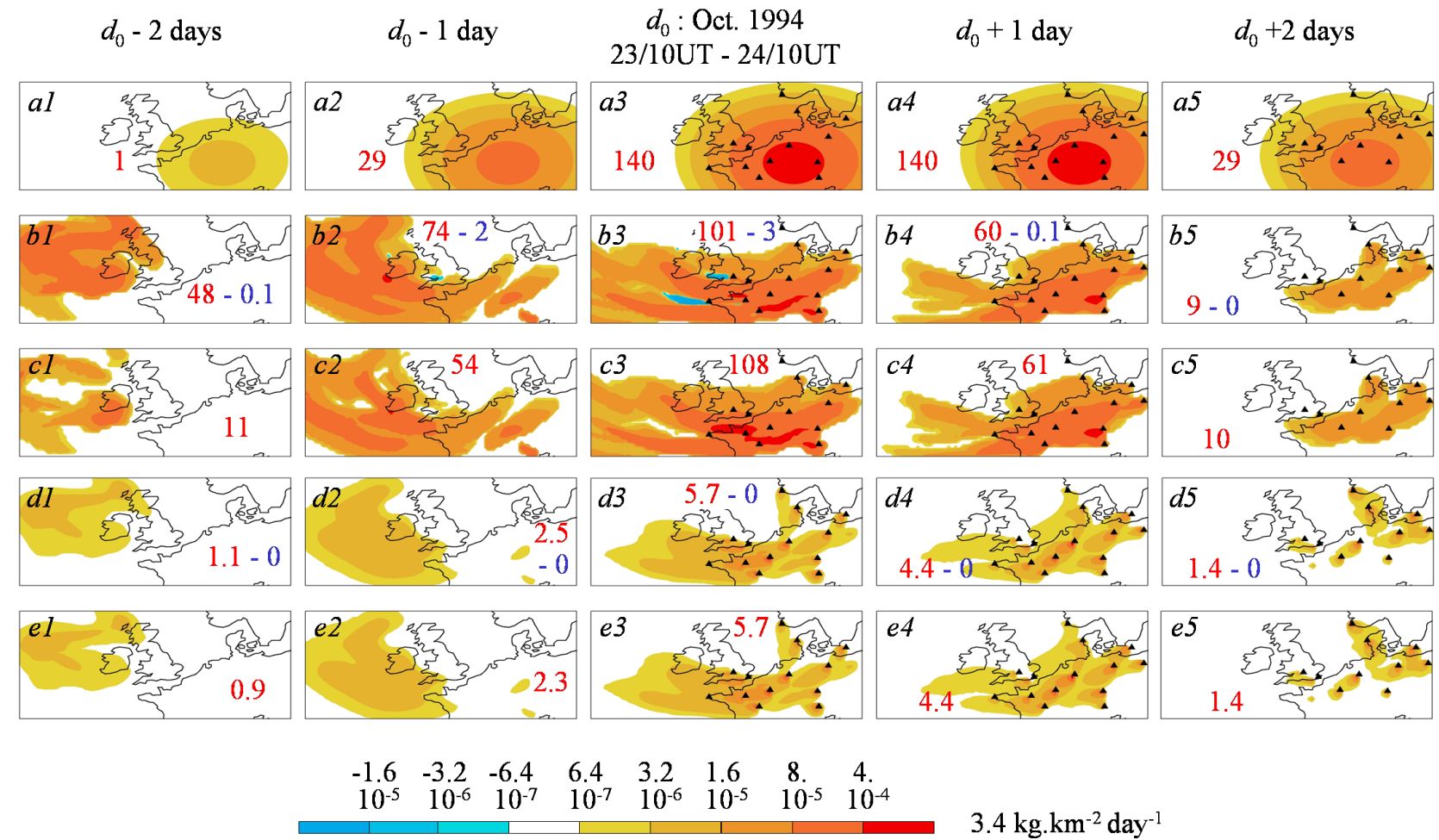

Fig. 10. Reconstruction, with meshes $0.5^{\circ} \times 0.5^{\circ}$, of a Gaussian surface source with renormalised adjoint functions corresponding to the selection of 137 measurements. The daily integrated evolution of the source to be rebuilt is represented in (a) for five successive $24 \mathrm{~h}$ periods. This source is centred at 24 Oct. 94, 10:00 UT, and at $8^{\circ} \mathrm{E}, 49^{\circ} \mathrm{N}$ in order to be well inserted among the measurements. The characteristic length and duration of this $340 \mathrm{~kg}$ Gaussian release were $167 \mathrm{~km}, 18 \mathrm{~h}$. In (b) and (c) the source is rebuilt out of renormalised retroplumes respectively without and with a positivity constraint. In (d) the non renormalised algebraic estimate includes negligible negative contributions, but is still lightly different from its positive analogue in (e) in the early poorly seen regions. The red and blue numbers indicate the total amount, in $\mathrm{kg}$, of the positive and negative releases during the successive periods with the active detectors indicated by triangles. The results should be compared to the illumination shown by the Fig. $4 \mathrm{~b}$.

stem error free from the unknown real source $\sigma$. Thus the future reality is made a statistical thing. The dotted statistics are a property of the observational setup, describing which values of the measurements could be expected to come out related to which observable sources. For lack of knowledge the average anticipation is $\dddot{\mu}=0$. The reading of the very observations $\boldsymbol{\mu}^{\text {obs }}$ closes the time of the suppositions as then we learn $\boldsymbol{\mu}=\boldsymbol{\mu}^{o b s}$. The anticipations, as a statistical interpretation of the renormalised geometry, do not correspond to the stage after the availability of the observations.

After the observations more classical statistics take over related to the technical quality of the setup. The corresponding averages are denoted with a solid bar. From $\mu$ that would be ideally observed, $\boldsymbol{\mu}^{o b s}$ stray with a error $\delta \boldsymbol{\mu}=\boldsymbol{\mu}^{o b s}-\boldsymbol{\mu}$. This error accounts for the known limitation of the detector quality and may also account for the generally not so well known limitation of the transport model representativity: $\delta \boldsymbol{\mu}=\delta \boldsymbol{\mu}_{d}+\delta \boldsymbol{\mu}_{t}$. If the error is unbiased, $\overline{\delta \boldsymbol{\mu}}=0$. Thus, once $\boldsymbol{\mu}^{o b s}$ has been read, the conditional expectation for the ideal $\boldsymbol{\mu}$ becomes: $\overline{\mu_{/ \mu^{o b s}}}=\boldsymbol{\mu}^{o b s}$. The rebuildable part of the source is observed with an error:

$$
\begin{array}{lc}
\sigma_{\| \varphi}^{o b s}={ }^{t} \boldsymbol{\mu}^{o b s} \mathbf{H}_{\varphi}^{-1} \boldsymbol{r}_{\varphi} & \delta \sigma_{\| \varphi}={ }^{t} \delta \boldsymbol{\mu} \mathbf{H}_{\varphi}^{-1} \boldsymbol{r}_{\varphi} \\
\overline{\delta \sigma_{\| \varphi}(\boldsymbol{x})}=0 & \mathbf{Q}=\overline{{ }^{t} \delta \boldsymbol{\mu} \delta \boldsymbol{\mu}} \\
\overline{\delta \sigma_{\| \varphi}(\boldsymbol{x}) \sigma_{\| \varphi}(\boldsymbol{y})}={ }^{t} \boldsymbol{r}_{\varphi}(\boldsymbol{x}) \mathbf{H}_{\varphi}^{-1} \mathbf{Q} \mathbf{H}_{\varphi}^{-1} \boldsymbol{r}_{\varphi}(\boldsymbol{y})
\end{array}
$$

This may be compared to the anticipation 28 rewritten:

$$
\widetilde{\sigma}_{\| \varphi}(\boldsymbol{x}) \sigma_{\| \varphi}(\boldsymbol{y})={ }^{t} \boldsymbol{r}_{\varphi}(\boldsymbol{y}) \mathbf{H}_{\varphi}^{-1} \boldsymbol{r}_{\varphi}(\boldsymbol{x})
$$

The Figs. $8 \mathrm{c}, 9 \mathrm{c}$ show the variance $\overline{\delta \sigma_{\| \varphi}(\boldsymbol{x})^{2}}$ of the synthetic reconstructions of ETEX1 for a total Gaussian uncertainty representing $30 \%$ of the synthetic measurements. This is twice as large as the $15 \%$ evaluated for the detector errors (Joint Res. Centre, 1998) but probably not sufficient for the error of representativity of the transport model. We also ignored the correlated behaviour of the representativity errors and handled the following diagonal covariance matrix:

$\mathbf{Q}=0.09\left[\begin{array}{lll}\mu_{1}{ }^{2} & & \\ & \cdot & \\ & \mu_{n}{ }^{2}\end{array}\right]$ 


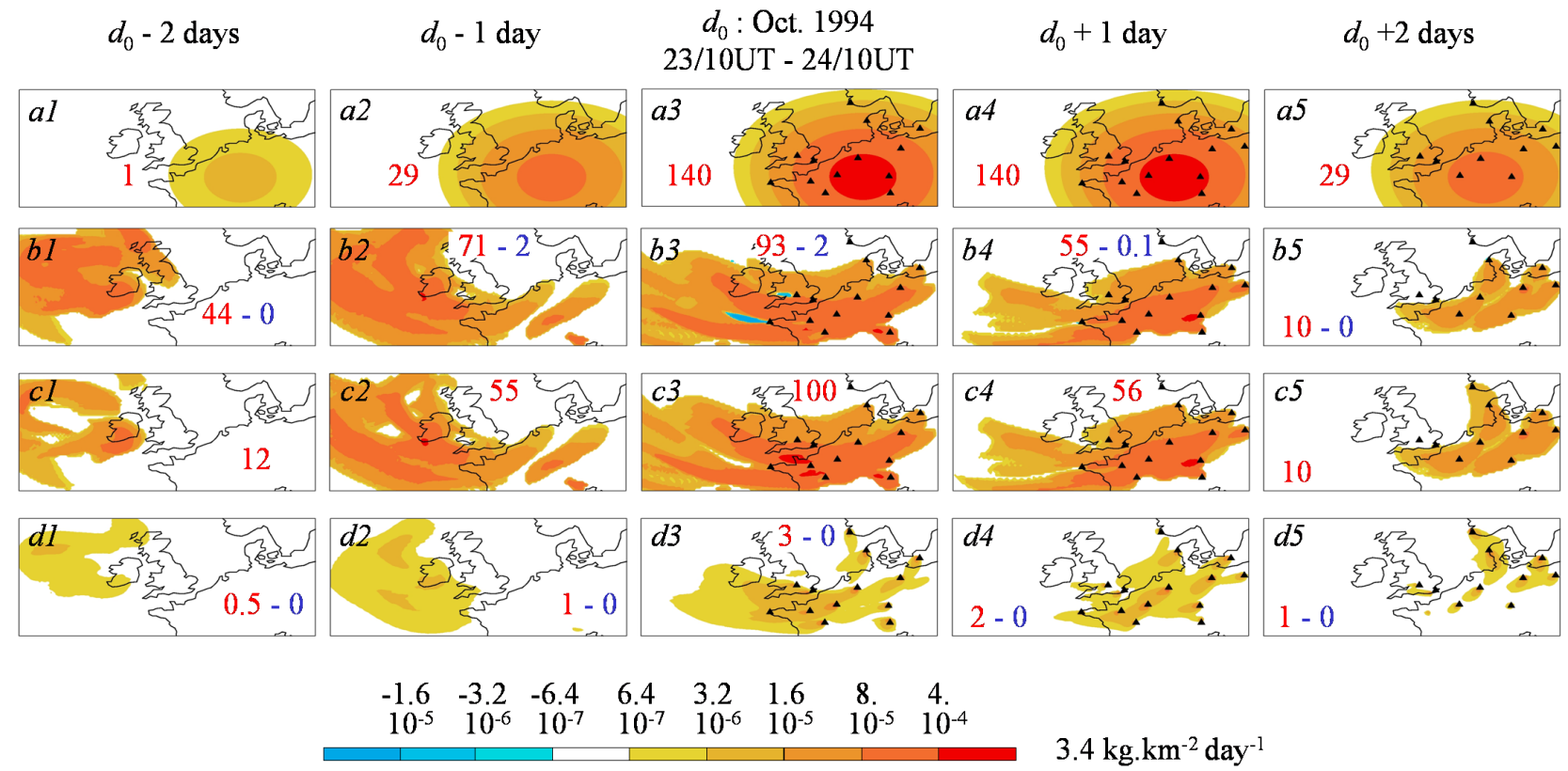

Fig. 11. Reconstruction of the same synthetic Gaussian source (a) as in the Fig. 10 with meshes $0.25^{\circ} \times 0.25^{\circ}$. The algebraic and positive reconstructions in (b) and (c) where renormalised retroplumes were used do not display important differences with the previous calculations using meshes $0.5^{\circ} \times 0.5^{\circ}$. On the contrary the non renormalised calculation in part (d) is very different: in the limit of an infinite resolution, not only the estimate of the source tends to zero but also Dirac detectors become uncorrelated. Therefore the algebraic reconstruction in (d) is already everywhere positive.

Note that the statistical law of the measurement errors may depend on the observations, here the synthetic $\mu_{i}$. The variance or covariance 38 are by no means an evaluation of $\sigma_{\perp \varphi}$. As already said in the Sect. 6, from the point of view of the monitoring network $\sigma_{\perp \varphi}$ is exactly as if it did not exist and this point of view would be ours if we could put all our information in the evaluation of $\sigma_{\| \varphi}$. The above variance and covariance just describe the uncertainty of the estimate stemming from the uncertainty of the measurements. It is accordingly natural to see that the variance evaluated on the Figs. 8 b3bis, 9 b3bis are not representative for the huge local difference between the ETEX1 point release and its estimate.

The same figures show weak errors in the regions poorly seen. In the regions not seen at all there is logically no error: there is no estimate. Though logical this result may upset the common idea that the quality of an estimation would be locally described by a local level of error. This quality is also tied to the sufficiency or insufficiency of the observations resulting in a necessary smoothness of the estimate. This local deficiency of information is not equivalent to an error which in fact was already said when stressing in the Sect. 6 the essentially unknown nature of the difference $\sigma_{\perp \varphi}=\sigma-\sigma_{\| \varphi}$. This behaviour is proper to the observation of infinite dimensional systems. For a finite dimensional system it would be possible to observe all the degrees of freedom and our knowledge would be limited only by the quality of the observations. Our sources are not completely knowable with a finite number of observations. Independently of the quality of these observations the infinite dimensionality still produces uncertainties described, we think, by the function $\varphi$. The comparison on the Figs. 6 and 7 of the results obtained for synthetic or real data shows that the smooth inaccuracy of the estimate is mainly due to the insufficiency of the data.

These ideas suggest that the complete identification of the usual background error covariance matrix $\mathbf{B}$ with the covariance matrix of the measurement anticipations $\mathbf{B}_{\varphi}$ could be mainly a matter of reinterpretation of the first one. This identification, if it could be further justified, would enable a complete computation of the matrix based on the position and date of the measurements together with the current meteorological conditions, instead of the usual a priori and a posteriori approximations. There would still be the following difference between the classical and present theories which suggest, for the future, to investigate carefully their statistical properties.

The observations $\boldsymbol{\mu}^{o b s}$ with their unbiased error $\delta \boldsymbol{\mu}$ are directly linearly transformed into an estimate $\sigma_{\| \varphi}^{o b s}$ with a zero mean error $\delta \sigma_{\| \varphi}$. In particular the estimate would produce measurements equal to the observations: $\left(\sigma_{\| \varphi}^{o b s}, r_{\varphi i}\right)_{\varphi}=\mu_{i}^{o b s}$. This is because, contrary to the classical theory, the information provided by the observations is not combined to an priori evaluation of the sought source: there is none. Here the a priori zero mean anticipation $\dddot{\mu}=0$, or $\dddot{\sigma}=0$, is not a model. It is not an information competing with $\boldsymbol{\mu}^{o b s}$. It is on 


$$
d_{0}-2 \text { days } \quad d_{0}-1 \text { day }
$$

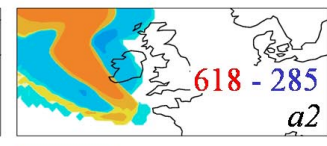

$d_{0}$ : oct. 1994

23/10UT - 24/10UT $d_{0}+1$ day
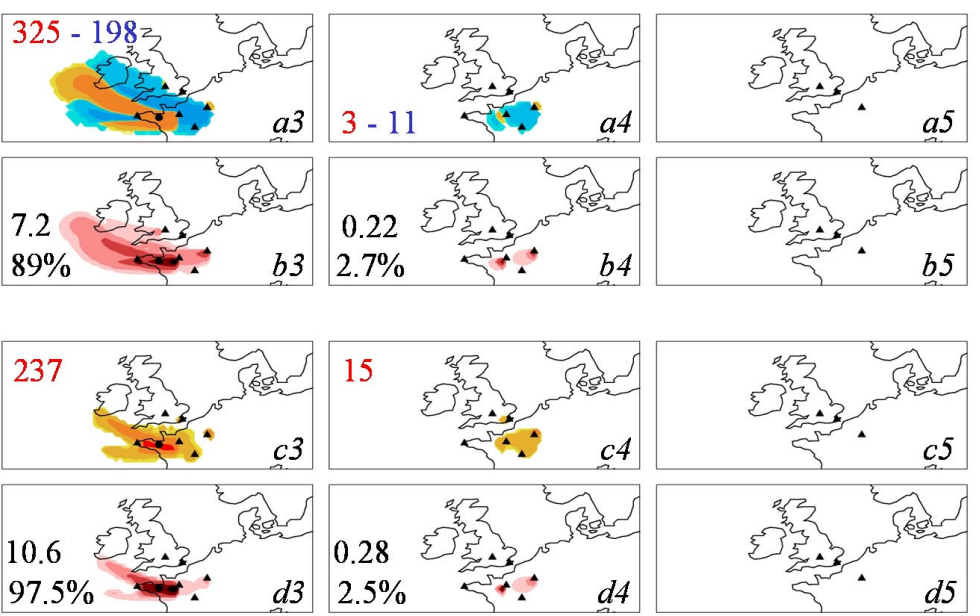

$d_{0}+2$ days

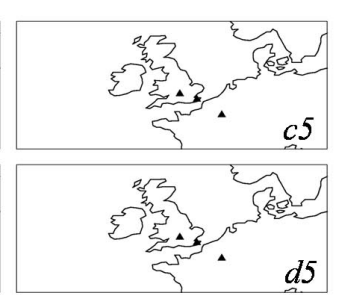
5
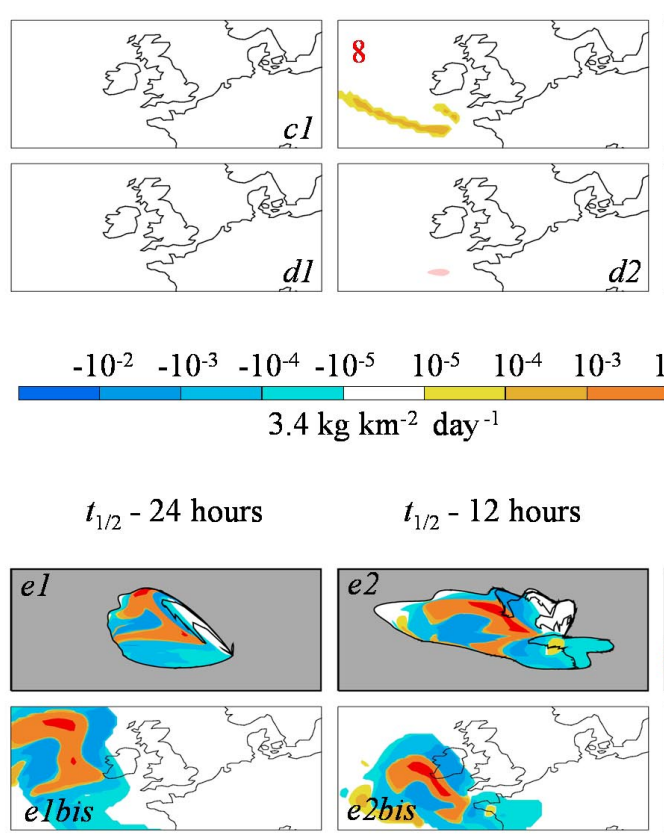

$3.4 \mathrm{~kg} \mathrm{~km}^{-2}$ day $^{-1}$

$$
t_{1 / 2}-12 \text { hours }
$$

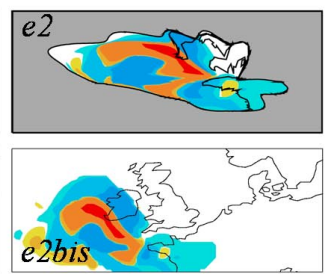

$t_{1 / 2}: 23 / 10 / 94,22 \mathrm{UT}$

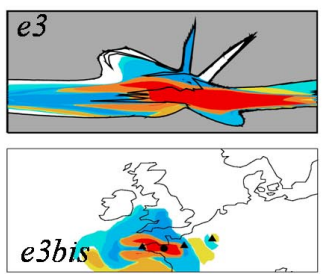

synthetic measurements

Fig. 12. During the calculation of the renormalising function $\varphi$, after the first iteration, the points $\boldsymbol{x}$ for which ${ }^{t} \boldsymbol{r}_{f_{0}}(\boldsymbol{x}) \mathbf{H}_{f_{0}}^{-1} \boldsymbol{r}_{f_{0}}(\boldsymbol{x})$ is less than a given threshold are removed from the iterations, and from the inversion, by prescribing $\varphi(\boldsymbol{x})=0$. The present reconstruction of ETEX1 for 51 synthetic measurement is analogous to the previous one except that the cutting threshold is 1000 times more little. More points are effectively considered. The parts (a), (b), (c), (d) of the figure should be compared to the Fig. 6a, b, c, d and its part e to the Fig. 8 a. The reconstruction looks somewhat different when the estimate is considered in the ordinary geometry. The difference vanishes in terms of the informational energy. In fact the weight of the additional points is weak. The renormalised geometry and $\varphi$ are almost unaltered and, when the source estimate is considered in this geometry, the changes are shown to be unimportant.

the contrary a default value of $\boldsymbol{\mu}^{o b s}$ for lack of any information until the reading. This zero says that nothing is known about $\sigma$ until the reading.

Through the minimisation of the functional $\mathcal{J}(s)$, Eq. 18, the classical theory balances the information obtained from the measurements against the information produced by an a priori model of the source. Let $\mathbf{H}_{b}=\mathbf{R B}{ }^{t} \mathbf{R}$. The classical estimate $\sigma^{e s t}$ produces measurements $\boldsymbol{\mu}^{\text {est }}=\left(\mathbf{I}+\mathbf{Q} \mathbf{H}_{b}^{-1}\right)^{-1} \boldsymbol{\mu}^{\text {obs }}$ generally distinct from $\boldsymbol{\mu}^{o b s}$; see for instance the deduction of Courtier (1997). Note that if we introduce in discrete matrix form the functions adjoint for $\langle,\rangle_{b}$ to the measurements, $r_{i}=\mathbf{B} r_{i}$, then $\mathbf{H}_{b}$ is the Gram covariance matrix of elements $\left\langle r_{b i}, r_{b j}\right\rangle_{b}={ }^{t} r_{b i} \mathbf{B}^{-1} r_{b j}={ }^{t} r_{i} \mathbf{B} r_{j}$.

\section{The informational energy}

The algebraic estimate may look disappointing on the Figs. 6, 7 , even based on synthetic data. Its structure around the very space time location of the ETEX1 release is correct consistently with the space time density of the measurements. Nevertheless the early contributions are excessive. The default, mostly improved by the positive estimation, is, we think, in the nature of the things, it must be understood and accepted. The figures provide representations in the ordinary geometry which is of an utmost convenience to us, but not to the monitoring network living in the geometry of $\langle,\rangle_{\varphi}$. The estimates $\sigma_{\| \varphi}, \sigma_{\varphi}^{+}$do belong to this geometry, not to the ordinary one. The simplest way to account for this is by representing them 
in the renormalised geometry, which is made, according to the explanations of the Sect. 10, on the Figs. 8, 9. There the previously unsatisfactory parts of the estimates, in fact associated to poorly illuminated regions, are much reduced.

These ideas enable to address the consequence of an improvement, i.e. a reduction, of the cutting threshold $\beta$ in the algorithm 12, 13. This would cause the effective involvement of more regions into the calculations. The reduction of a $\beta$ already small enough would just add marginally seen regions having a marginal importance. The Fig. 12 shows results obtained for a reduction of $\beta$ by a factor of 1000 compared to the Figs. 6 and 8. The respective estimates look different when they are represented in the ordinary geometry. Their equivalence appears in the renormalised geometry, itself practically unaltered.

This discussion finally leads to the conclusion that it is inappropriate to consider such a total release as $\int_{\Omega \times \mathrm{T}} \rho \sigma_{\| \varphi}(\boldsymbol{x}) d \boldsymbol{x}$. This would amount to adding well and poorly seen contributions. The integral might as well be divergent. It is probably possible to consider total releases integrated for a limited subdomain having a uniformly good illumination, but the very meaning of this should be further investigated. We think that the only appropriate global integral quantity is $\int_{\Omega \times \mathrm{T}} \rho \varphi \sigma_{\| \varphi}^{2} d \boldsymbol{x}$. This leads now to the definition of an informational energy.

The norm of a source was seen to decompose as:

$$
\begin{aligned}
(\sigma, \sigma)_{\varphi} & =\int \rho \varphi \sigma^{2} d \boldsymbol{x}=\langle\sigma, \sigma\rangle_{\varphi}+\left(\sigma_{\perp \varphi}, \sigma_{\perp \varphi}\right)_{\varphi} \\
\langle\sigma, \sigma\rangle_{\varphi} & =\left(\sigma_{\| \varphi}, \sigma_{\| \varphi}\right)_{\varphi}
\end{aligned}
$$

Indirectly $(\sigma, \sigma)_{\varphi}$ is a measure of the amount of tracer released but not only. The total release may even vanish if the positive and negative contributions compensate. Thus $(\sigma, \sigma)_{\varphi}$ is also a measure of the structural complexity of the source. The part of this complexity that may be captured by the detectors is $\langle\sigma, \sigma\rangle_{\varphi} \leq(\sigma, \sigma)_{\varphi}$. From the point of view of the detectors handled with the renormalised geometry, $\sigma_{\| \varphi}$ is $\sigma$ and $\langle,\rangle_{\varphi}$ is $(,)_{\varphi}$. Nevertheless the theoretician often puts himself in the situation to know more about a source than the information available from the measurements. For him $(,)_{\varphi}$ and $\langle,\rangle_{\varphi}$ are different. For instance we know the ETEX1 release but pretend in our calculations that we know only a few measurements. We propose to use in this situation the following wording. The quadratic form $(\sigma, \sigma)_{\varphi}$ will be called the informational energy of the source $\sigma$ and we shall say that $\langle\sigma, \sigma\rangle_{\varphi}=\left(\sigma_{\| \varphi}, \sigma_{\| \varphi}\right)_{\varphi}$ is the part of this energy captured by or interacting with the detectors. Then $\varphi(x) \sigma(x)^{2}$ will be the density of energy per unit mass around the space time position $\boldsymbol{x}$ in the ordinary geometry where the elementary mass is $\rho d \boldsymbol{x}$. In the renormalised geometry, the element is $\rho \varphi d \boldsymbol{x}$ and the density of informational energy is simply $\sigma(x)^{2}$. So, contrary to $\sigma_{\| \varphi}(\boldsymbol{x})$, the new quantity undergoes natural transformations according to the geometry chosen for its representation. Its interest is shown in the ordinary geometry by the Figs. 6, 7, in the renormalised geometry by the Figs. 8 , 9. The density of informational energy captured by the monitoring network, especially in the positive case, is unambiguously focused in space and time by the position of the point release ETEX1.

The present theory seems to display links with theoretical physics. The entropic constraints 32 may be indeed related, according to how it is looked at, either to the general relativity, or to the quantum theory.

The criterion, in its form $E_{\varphi}(\boldsymbol{x})=\varphi(\boldsymbol{x})$, involves $\varphi$ defined as a geometric weight or mass attributed to the ordinary geometry while its illumination $E_{\varphi}(\boldsymbol{x})=\varphi(\boldsymbol{x})^{t} \boldsymbol{r}_{\varphi}(\boldsymbol{x}) \mathbf{H}_{\varphi}^{-1} \boldsymbol{r}_{\varphi}(\boldsymbol{x})$ behaves like a density of energy. This is an evocation of the general relativity, $E=m c^{2}$, relying on interpretations of $E_{\varphi}$ and $\varphi$ to be supported by further investigations probably based on tensor analysis.

The link with the quantum theory was already suggested by the discussion at the end of the Sect. 7. The following notations will be convenient to better see the relevance of the comparison. To any position $\boldsymbol{x}$ and any source $\sigma$, producing measurements $\boldsymbol{\mu}$, we associate the following vectors in $\mathbb{R}^{n}$ with a double notation as standard vectors and a quantum notation as "bras" or "kets":

$$
\begin{aligned}
& \hat{\boldsymbol{r}}(\boldsymbol{x})=|\boldsymbol{x}\rangle=\sqrt{\mathbf{H}_{\varphi}^{-1}} \boldsymbol{r}_{\varphi}(\boldsymbol{x}) \quad\langle\boldsymbol{x}|={ }^{t} \hat{\boldsymbol{r}}(\boldsymbol{x}) \\
& \hat{\boldsymbol{\mu}}=|\sigma\rangle=\sqrt{\mathbf{H}_{\varphi}^{-1} \boldsymbol{\mu}} \quad\langle\sigma|={ }^{t} \hat{\boldsymbol{\mu}} \quad \ldots \ldots \ldots . \\
& \hat{\boldsymbol{\mu}}
\end{aligned}
$$

This linear recombination enables to have the identity matrix as the covariance matrix of the measurement anticipations. Then we obtain for sources $\sigma, \sigma^{\prime}$ producing measurements $\boldsymbol{\mu}, \boldsymbol{\mu}^{\prime}$, and for space time positions $\boldsymbol{x}, \boldsymbol{y}$ :

$$
\begin{aligned}
& \left\langle\sigma, \sigma^{\prime}\right\rangle_{\varphi}={ }^{t} \boldsymbol{\mu} \mathbf{H}_{\varphi}^{-1} \boldsymbol{\mu}^{\prime}={ }^{t} \hat{\boldsymbol{\mu}} \hat{\boldsymbol{\mu}}^{\prime}=\left\langle\sigma \mid \sigma^{\prime}\right\rangle \\
& { }^{t} \boldsymbol{r}_{\varphi}(\boldsymbol{x}) \mathbf{H}_{\varphi}^{-1} \boldsymbol{r}_{\varphi}(\boldsymbol{y})={ }^{t} \hat{\boldsymbol{r}}(\boldsymbol{x}) \hat{\boldsymbol{r}}(\boldsymbol{y})=\langle\boldsymbol{x} \mid \boldsymbol{y}\rangle \\
& { }^{{ }_{\| \varphi}}(\boldsymbol{x})={ }^{t} \boldsymbol{\mu} \mathbf{H}_{\varphi}^{-1} \boldsymbol{r}_{\varphi}(\boldsymbol{x})={ }^{t} \hat{\boldsymbol{\mu}} \hat{\boldsymbol{r}}(\boldsymbol{x})=\langle\sigma \mid \boldsymbol{x}\rangle
\end{aligned}
$$

The pivotal point is the form taken by the entropic constraint 32 for a positional vector:

$$
\langle\boldsymbol{x} \mid \boldsymbol{x}\rangle=1
$$

The recombined vectors $\hat{\boldsymbol{r}}(\boldsymbol{x})=|\boldsymbol{x}\rangle$ are thus analogous to the pure states of a quantum system. This supports the interpretation of the renormalisation as an equipartition of the information. A reader not familiar with this theory may consult the presentations by Kholevo (1980) or Fano (1967). It is now justified to investigate more accurately the connections between data assimilation and the statistical formalism of the quantum theory. If a vector $\hat{\boldsymbol{r}}(\boldsymbol{x})=|\boldsymbol{x}\rangle$ is considered as a source $\sigma_{\boldsymbol{x}}(\boldsymbol{y})=^{t} \hat{\boldsymbol{r}}(\boldsymbol{x}) \hat{\boldsymbol{r}}(\boldsymbol{y})$, the integral form of its norm 44 becomes:

$$
\int\langle\boldsymbol{x} \mid \boldsymbol{y}\rangle^{2} \rho \varphi(\boldsymbol{y}) d \boldsymbol{y}=1
$$


Hence the density of informational energy $\langle\boldsymbol{x} \mid \boldsymbol{y}\rangle^{2}$ of the source $|\boldsymbol{x}\rangle$ at the position $\hat{\boldsymbol{r}}(\boldsymbol{y})=|\boldsymbol{y}\rangle$ might as well be regarded as probability distribution for the unit amount of energy $\langle\boldsymbol{x} \mid \boldsymbol{x}\rangle=1$. Accordingly $|\boldsymbol{x}\rangle$ behaves like a wave function for this energy. This would account for the impossibility to retrieve a point release at the position $\boldsymbol{x}$ with an infinite resolution.

\section{The known domain}

The transformation $\boldsymbol{x} \mapsto|\boldsymbol{x}\rangle=\hat{\boldsymbol{r}}(\boldsymbol{x})$ may be regarded as a mapping or coordinate change sending $\Omega \times \mathrm{T}$, onto a fourdimensional submanifold $\mathcal{C}$ of the unit sphere $\mathbb{S}^{n}$ of $\mathbb{R}^{n}$. This submanifold, endowed with its ordinary Euclidean geometry, gives the best natural account of the information: no renormalisation is necessary for it.

Indeed as a result of the renormalisation the probability distribution 30 is constant for all vectors $\hat{\boldsymbol{\mu}} \in \mathbb{S}^{n}$, $q_{\varphi}(\hat{\boldsymbol{\mu}})=(2 \pi)^{-\frac{n}{2}}$ which means that the parts of $\mathbb{S}^{n}$ are measured proportionally to their (n-1)-dimensional Euclidean volume. As a submanifold of $\mathbb{S}^{n}, \mathcal{C}$ is endowed with the 4-dimensional Euclidean geometry inherited from the (n-1)-dimensional Euclidean geometry of $\mathbb{S}^{n}$. This implies that the Jacobian function $g$ of the transformation, $d \boldsymbol{x}=g(\hat{\boldsymbol{r}}) d \hat{\boldsymbol{r}}$, is equal to $\varphi^{-1}$ in order to transform the measurement $\hat{\mu}_{i}=\int_{\Omega \times \mathrm{T}} \sigma \hat{r}_{i} \varphi \rho d \boldsymbol{x}$ into the Euclidean integral: $\hat{\mu}_{i}=\int_{\mathcal{C}} \sigma \hat{r}_{i} \rho d \hat{\boldsymbol{r}}$.

The geometry of $\mathcal{C}$ cannot be represented on a sheet of paper. The main problem is that $\mathcal{C}$ is embedded in $\mathbb{R}^{n}$. This geometry was obtained from the ordinary geometry by attributing the point $x$ of the ordinary domain $\Omega \times \mathrm{T}$ a weight $\varphi(\boldsymbol{x})$. It is possible to obtain a satisfactory account of this selected property. We just have to find some mapping from $\mathbb{R}^{4}$ into itself with $\varphi$ as Jacobian function. The Figs. 4, 5, 8, 9 and 12 were built as follows. As the source was investigated at the surface $\Sigma$ of the ground or oceans, the working domain was in fact $\Sigma \times T$. Then, for some selected moment $t_{0}$ we obtained an illumination $\varphi\left(x, y, t_{0}\right)$. For the Figs. 4 and 5 the illumination was averaged on $24 \mathrm{~h}$ periods. It was then very easy to obtain a two-dimensional transformation of $\Sigma$ with the given Jacobian $\varphi\left(x, y, t_{0}\right)$. To this end the Cartesian coordinates $(x, y)$ are first replaced by polar coordinates $(l, \theta)$ for some given centre. Then, with $\theta$ kept unchanged, $l$ is transformed into $l^{\prime}$ according to the following differential equation which provides the adequate transformation of the surface and time element:

$d l^{\prime 2}=\varphi\left(l, \theta, t_{0}\right) d l^{2}$

$d \theta l^{\prime} d l^{\prime} d t=\varphi\left(l, \theta, t_{0}\right) d \theta l d l d t$

The representation obtained this way is just an approximation of the known domain but it emphasises several points.

Firstly, the known domain is finite. Its total weight is the number of measurements. On the distorted maps obtained for Europe on the Figs. 4, 5, 8, 9, 12 an edge or horizon is clearly visible.

Secondly the illumination is important in the space-time neighbourhood of the detectors. This produces flare shaped excrescences on the figures. The detailed behaviour cannot be investigated by numerical tools; we think that $\varphi$ should display a square summable singularity by the point detectors. No flare is visible on the subfigures 4 albis, a2bis, blbis, b2bis corresponding to periods of time with no active detector. In the very geometry of $\mathcal{C}$ each flare corresponds to a deep cone with, locally, a symmetric arrangement around a detector in the bottom of the cone.

Thirdly there is an intermediate region between the "deep" detectors and the remote horizon. In this region the distortion is not so big and the European coastline is well identifiable.

Fourthly the regions away from the detectors become smaller and smaller. We think this corresponds to the well known conic illusion that a remote object looks smaller. The coast of Iceland, of the Iberian peninsula, of Scandinavia finally become chaotic zigzags along the horizon. This is illustrated by the Fig. 5. A source of tracer there will be more and more difficult to capture with the measurements.

The approximate representation for the known domain puts all the distortion on the space coordinates $(x, y)$. It should be noted that in the finite geometry of $\mathcal{C}$ the distortion with respect to the ordinary geometry necessarily touches $t$ as well. The following arguments even suggest a severe contraction of the time close to the horizon. The measurements performed for ETEX cover a period of time of $90 \mathrm{~h}$. The adjoint functions $r_{i}$ of these measurements behave like retroplumes transported back in time by the winds and scattered by the diffusion. Due to the diffusion, the regions far away will have been passed over by the retroplumes during periods of time all the larger than the original $90 \mathrm{~h}$ as they lie further away from the monitoring network. This means in turn that the $90 \mathrm{~h}$ measurements will have captured a part of existence of these remote regions representing more, possibly much more, than $90 \mathrm{~h}$. Just as if, by sitting in the countryside for one hour and looking far enough, we could see a tree growing from a seed, have fruits and die. One may for instance consider that, at times preceding the states represented on Fig. 4a1, b1, the illumination extends through the Atlantic ocean to North America. At these times the retroplumes are so flattened and homogenised that their ability to discriminate the structural details of a source there and then is purely residual. Accordingly the contribution to the known domain by the long passing of the adjoint functions above this continent should reduce to a tiny spot. Due to the scarcity of the information in the space-time regions close to the horizon, the very structure of a source there is lost in practice. The image of the growing tree was idealised: with the logical connections mostly cancelled by the scarcity of the information, we would rather see a chaotic agitation of seeds, fruits and branches. 


\section{Results}

obtained from the calculations described in the Sect. 4 for the estimation of the source ETEX1 may be summarised as follows. The Fig. 2 is a comparison of the results obtained with the ordinary geometry and with a geometry transformed by the optimal renormalising function. The adjoint functions $r_{i}$ were calculated with a horizontal resolution of $0.5^{\circ} \times 0.5^{\circ}$. The illumination, algebraic and positive estimates are integrated in time for both selections of synthetic measurements, without or with renormalisation. The comparison of the subfigures 2 a1 and b1 or $\mathrm{c} 1$ and $\mathrm{d} 1$ shows a much better and wider distribution of the illumination in the renormalised case. Contrary to our expectation, the optimised illumination is not flat around the monitoring network. It still displays peaks, of a lesser intensity, around the detectors. The quality of the renormalised estimates is clearly improved. The maximum releases are no longer obtained by the positions of the detectors, they are found close to Monterfil. The magnitude of the total amount is now correct, closer to $340 \mathrm{~kg}$ in the positive case. The Fig. 3 corresponds to the same description except that the calculation of the $r_{i}$ and the inversion were performed with a higher horizontal resolution of $0.25^{\circ} \times 0.25^{\circ}$. The meteorological fields were simply interpolated and the inversion was based on the same synthetic measurements produced by the coarsest resolution. The renormalised results display a noticeable stability with respect to the improvement of the resolution. On the contrary the non renormalised inversion is clearly more focused around the detectors with surrounding releases decreased by at least a factor two.

The Fig. 4, prepared in resolution $0.5^{\circ}$, illustrates the geometric meaning of the optimal renormalising function $\varphi$ coinciding with its own illumination. The time evolution of the known domain $\mathcal{C}$ is represented for five successive days by the flat approximations corresponding to a radial transformation centred at Monterfil, except for the subfigure $4 \mathrm{b5ter}$. The known domain is finite, limited by a space time horizon in red on the figures. The effect of aperture from the subfigures 4 albis to a3bis or blbis to b3bis illustrates as well its finite extent in the past. The known domain is finite towards the future, nothing is known after the end of the last measurement. This shutting is visible on the subfigure 4 a5bis. The comparison of the parts $\mathrm{a}$ and $\mathrm{b}$ illustrates the influence of a greater number of measurements. In particular the second selection includes many measurements for the fifth period and the known domain is still wide open. At that moment the illuminated cloud is shifted to the north east and a radial transformation centred in the Danish islands produces on the subfigure $4 \mathrm{~b} 5$ ter lesser distortions than may be seen in b5bis.

The finite extension of the known domain does not mean that finitely many regions are seen. It means that large, possibly infinitely large remote regions of the space time domain will be attributed a residual information weight. As an illustration Iceland is reduced on the Fig. 5 to a thin zigzag along the horizon, close to Ireland.

The quality of the algebraic sources rebuilt on the Figs. 6 and 7 is better for the periods including and following the real twelve hour release. As already shown by the Fig. 4 the region of Monterfil is better seen during the period after the release than before. The tracer contribution from the early poorly seen regions is overestimated. The overestimation of the first two periods could mean that there is not enough information in the selections of measurements to decide between a smaller close source or a bigger one further away. This interpretation may be compared to the impossibility to estimate the distances and the real dimensions of the stars with the naked eyes. A valuable correction is brought by the positivity constraint. The representation of the informational energy displays a surprisingly well peaked structure by the space time position of the very release. This may be understood because in the ordinary geometry the density of energy $\varphi \sigma^{2}$ is a compromise including the renormalising or illumination function which is weak during the first two periods. The comparison of the Figs. 6 and 7 mainly shows that, due to a greater number of measurements the reconstruction from the real data is subject to more model errors. These are seen on the subfigures $\mathrm{f} 4, \mathrm{f} 5, \mathrm{~h} 4, \mathrm{~h} 5$ in the form of peaks of energy by the positions of the detectors.

For the synthetic inversions the focus of the energy in the appropriate part of the known domain is even clearer in the renormalised geometry of the Figs. 8, 9, part b. Note that the total duration covered by these figures is 48 hours instead of 120 for the previous ones. The comparison of the subfigures $8 b 3, b 4,9 b 3, b 4$ indicate a better focus in time for the selection of 51 measurements and in space for the selection of 137 measurements. The most interesting comparison is between the part $c$ of the figures describing the variance of the estimate as a result of measurement errors representing $30 \%$ of the signal. The variance associated to 137 measurements is roughly three time less than the variance associated to 51 measurements. In both cases the variance is one order of magnitude below the informational energy. This suggests that the difference shown by the Figs. 6 and 7 between the sources rebuilt out of synthetic or real measurements is due mostly to the model errors.

The algebraic and positive reconstructions of a widespread source on the Fig. 10 for a resolution $0.5^{\circ}$ and on the Fig. 11 for $0.25^{\circ}$ support the above conclusions. The non renormalised inversion is considerably changed, more focused around the detectors for the thinnest resolution. The algebraic estimate is essentially positive and coincides with the positive one almost in the coarse case and completely in the thin case. This is not a good indication: it means that the correlations between the measurements are ignored. The comparison shows again the stability of the renormalised inversion. The general features of the source are recovered, the total amount of the release for each period is retrieved within a factor two. There is still an overestimation of the early releases, when the illumination is low. Again this aberration 

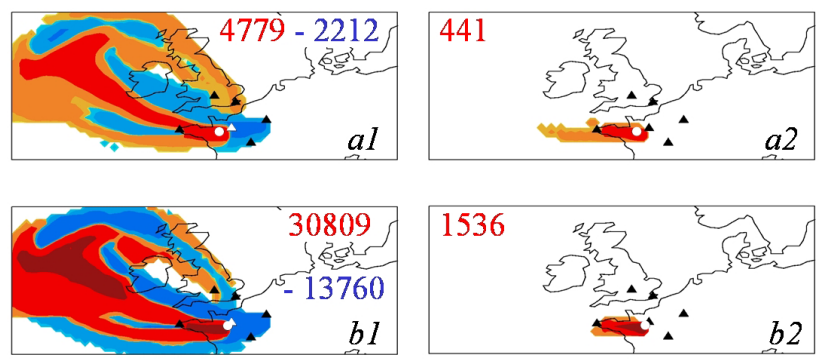

$\begin{array}{lllllll}-10^{-2} & -10^{-3} & -10^{-4} & 10^{-4} & 10^{-3} & 10^{-2} & 10^{-1}\end{array}$

$3.4 \mathrm{~kg} \cdot \mathrm{km}^{-2}$

Fig. 13. The source ETEX1 is located in Monterfil, $163 \mathrm{~km}$ or five meshes of $0.5^{\circ}$, west of the station F2 in Alençon. The figure represents the time integrated algebraic and positive inversions for the 51 synthetic measurements produced by a source of $340 \mathrm{~kg}$ (a): $65 \mathrm{~km}$ or two meshes west of Alençon, (b): in Alençon. These releases are still spread on $12 \mathrm{~h}$ starting from the 23 October 1994, 16:00 UT. For a synthetic source in Monterfil the four measurements by F2 during these 12 hours would have an average value of $2.7 \mathrm{ng} . \mathrm{m}^{-3}$. This value goes up to $15.4 \mathrm{ng} \cdot \mathrm{m}^{-3}$ in the case (a) and to $99.3 \mathrm{ng} \cdot \mathrm{m}^{-3}$ in the case (b). The other detectors are not significantly affected. The red and blue numbers indicate the total amounts, in $\mathrm{kg}$, of the positive and negative estimated releases.

is mostly corrected by the positivity constraint. It must be noted that the quality of the estimate is better in the close environment of the active detectors. This is very visible with the deep low valued indentation tied to the station F8, Brest, on the subfigures $\mathrm{c} 3, \mathrm{c} 4, \mathrm{~d} 3, \mathrm{~d} 4$.

The Fig. 12 emphasises the meaning of the theory. It shows the same synthetic calculations tied to 51 measurements as previously the Figs. 6 and 8. Nevertheless in the implementation of the condition 12 the cutting threshold $\beta$ is reduced by a factor 1000 so that some additional poorly seen regions are effectively included in the inversion. In the ordinary geometry the important differences of the algebraic estimates observed on the Figs. 6a, 12a completely vanish in terms of informational energy on the Figs. 6b, 12b. The comparison of the Figs. $8 \mathrm{a}$ and $12 \mathrm{e}$ shows a lack of consequences of the reduction of $\beta$ on the following two points. Firstly the renormalised geometry is not significantly altered. Secondly, when they are considered in the renormalised geometry, the algebraic estimates no longer display significant differences.

The Fig. 13 explores the effect of a point source close to a detector. When the source is put nearer and nearer the estimation becomes clearly divergent. In fact, when the source is put inside the detector, it is not the inversion which is divergent, it is the value of the measurements to which the estimation is linearly tied: $\sigma^{e s t}=\sum \mu_{i} g^{i}$ for some $g^{i}(\boldsymbol{x})$ with $\left(r_{i}, g^{j}\right)=\delta_{i}^{j}$ (Kronecker's symbols). A point source inside the detector is a highly "improbable" configuration not included in the optimally smooth inversion compromise. When the inversion is adequately smoothed the source is investigated at space and time scales consistent with the relative arrangement of the detectors. This means that if the distance between two detectors is typically $1000 \mathrm{~km}$ then any set of measurements will be interpreted in terms of a source at the continental scale with all other details smoothed as irrelevant. Then, if somebody scratches a match just in front of a detector, we shall logically conclude that the Amazonia is burning! Indeed the presence of an important point source much closer to a detector than the typical distance between two detectors is a serious disturbance and should be avoided.

\section{Discussion and perspectives}

The inverse technique proposed in this paper processes at once all the measurements available at all times in order to retrieve a source $\sigma_{\| f}$ with its complete time evolution. In this respect, it could be classified four-dimensional variational assimilation (Bouttier and Courtier, 1999). The evaluation of the estimation quality follows nevertheless an unusual scheme resulting from the distinction proposed between the measurement anticipations and the measurement errors. This evaluation is illustrated by the Figs. 8, 9. The simultaneous assimilation of all the data may also be regarded as a new feature. In classical assimilation the data are divided according to successive dates or periods and assimilated sequentially with a parallel improvement of the background error covariance matrix. In the present theory the measurements are jointly processed because they are all correlated as described by a background matrix of anticipations calculated from their positions and dates and from the meteorological conditions. An additional measurement obtained days later will not only be correlated with the measurements previously available, but also will change the correlations between the previous measurements. The assimilation will be non linearly altered and improved. Though logical this attitude will lead to a problem when the number of observations will be daily increased by the operation of a regular network. The difficulty may probably be solved reasonably as the correlation between two measurements becomes negligible if they are separated by a long delay. The results presented here show it is worth trying.

The calculation time required by the present method is probably larger, but reasonably, than required by a more traditional approach. Anyway the most costly stage is the preliminary calculation of the adjoint functions. As for the inversion itself, the time is roughly multiplied by the number of iterations towards the renormalising function as each require the calculation of one covariance matrix of size the squared number of measurements. The requirement that all the measurements be processed together has just been discussed. In compensation the method enables to describe wich regions should be really concerned by the inversion. This will provide a criterion to reduce considerably the calculations to the most relevant meshes in space or time. 
The comparison with the usual assimilation is so far incomplete because it is uneasy to compare the underlying different logics. The classical theory is organised as the quest of a source considered both real and unknown. In the present framework there is no further reality than the measurements and the meteorological fields. The quality of the estimation is limited in terms of smoothness and fuzziness by the availability of the information. This limitation is not an error because it does not represent a lag between the estimation and the reality: the estimation is the reality. The debate is not purely theoretical, the calculations presented here showed that the quality of the estimate was limited by the availability of the information more than by $30 \%$ of errors. The use of real data, containing both measurement and model errors, did not considerably degrade the synthetic inversion. This suggests that the errors are not preponderant in limiting the quality of the estimation. A numerical comparison of the theories, not done here, should certainly shed a useful light. This extreme point of view that the estimation is the reality is consistent, but the ways to relax it ought to be investigated. The a priori information that the source is positive was seen to considerably improve the estimate. Other a priori information might be included in the usual framework based on Kalman's filtering (Rödenbeck et al., 2003; Anderson and Moore, 1979), not yet in ours: the source lies rather here than there, varies slowly in space or time, is periodic.

Another difference is the absence in the present framework of a model of the source. Hence the information obtained from the measurements is not balanced with that from such a model. In particular the a priori expectation, called here anticipation, that the observations and the observed source should vanish in average, is not considered a piece of information. On the contrary, it is considered an absence of information, absence cancelled by the measurements when the results are known.

The definition of a "known domain" is both natural and useful. It is natural because the intuition perfectly agrees that the relevance of a set of data cannot extend indefinitely. And it is a useful diagnostics about the sufficiency and adequacy of a monitoring network. The known domain is a strange object. The measurements are combined to produce an estimate regardless of the precedence of the ones compared to the others. The idea of a time flying seems to vanish. In the known domain what would be the meaning of a ket $|\boldsymbol{x}\rangle$ 'before' a ket $|\boldsymbol{y}\rangle$ ? Keys would probably arise from an investigation of the form taken by the transport equation of the tracer after the transformation from the ordinary geometry. A transport equation should also be established for the informational energy. These investigations will require a better representation of the known domain than the above flat radial transformations. It seems that the complete description of its four-dimensional geometry embedded in a higher dimension is a matter of tensor analysis (Raschewski, 1953). This would enable to further explore the aforementioned analogies with the theoretical physics, the general relativity and quantum theory. In order to proceed more easily we neglected to introduce dimensional constants in the identification 27 of $(,)_{f}$ with $\langle,\rangle_{b f}$ and in the formula 35 for the entropy with the evaluation of a logarithm for $\operatorname{det} \mathbf{H}_{f}$. This does not alter the logics of the paper, except that it is natural in physics to understand the meaning of such constants with their logics and consequences.

It must be noted that the increasing development of non linear chemistry transport models (Mallet and Sportisse, 2004; Quélo, 2004) is a field of application of inverse techniques, in the hypothesis of linear perturbations. In the case of the present strategy, this would mean that the link is linear between the expectable mismatch of the sought source compared to an a priori estimate on the one hand and on the other hand the expectable mismatch of the measurements compared to their a priori values.

The word "renormalisation" was chosen by analogy with the theory of quantum electrodynamics where it designates the removal of an unphysical infinity by means of an infinite additive correction.

\section{A: Least entropy renormalisation}

We want to prove that, under the requirements $31, f(\boldsymbol{x})>0$, $\int_{\Omega \times \mathrm{T}} \rho f d \boldsymbol{x}=n$, the condition $32,{ }^{t} \boldsymbol{r}_{f}(\boldsymbol{x}) \mathbf{H}_{f}^{-1} \boldsymbol{r}_{f}(\boldsymbol{x}) \equiv 1$ is equivalent to minimising $\operatorname{det} \mathbf{H}_{f}$. We shall prove too that the function $\operatorname{det} \mathbf{H}_{f}$ is strictly convex among the acceptable $f$ so that it reaches a global minimum previously denoted $\varphi$.

Let's discretise the domain $\Omega \times T$ into a number $v_{\max }$ of meshes, like in the Sects .5, 6, with now the following notations: $\boldsymbol{x}^{v}$ and $d^{v}$ are the space-time position and space-time mass (the mass multiplied by the duration) of the meshes, $r_{i}^{\nu}=r_{i}\left(\boldsymbol{x}^{\nu}\right), f^{\nu}=f\left(\boldsymbol{x}^{\nu}\right), r_{f, i}^{\nu}=\frac{r_{i}\left(\boldsymbol{x}^{\nu}\right)}{f\left(\boldsymbol{x}^{\nu}\right)}$ etc. The discretisation can be made as thin as possible so that the results are valid for the continuous problem. With these notations the matrix $\mathbf{H}_{f}$ may be written:

$\mathbf{H}_{f}=$

$$
\left[\begin{array}{cc}
d^{1} \frac{r_{1}^{1} r_{1}^{1}}{f^{1}}+d^{2} \frac{r_{1}^{2} r_{1}^{2}}{f^{2}}+. ., & d^{1} \frac{r_{1}^{1} r_{2}^{1}}{f^{1}}+d^{2} \frac{r_{1}^{2} r_{2}^{2}}{f^{2}}+\ldots, \ldots \ldots . \\
d^{1} \frac{r_{2}^{1} r_{1}^{1}}{f^{1}}+d^{2} \frac{r_{2}^{2} r_{1}^{2}}{f^{2}}+. ., & d^{1} \frac{r_{2}^{1} r_{2}^{1}}{f^{1}}+d^{2} \frac{r_{2}^{2} r_{2}^{2}}{f^{2}}+. ., \ldots \ldots . \\
d^{1} \frac{r_{3}^{1} r_{1}^{1}}{f^{1}}+d^{2} \frac{r_{3}^{2} r_{1}^{2}}{f^{2}}+. ., & d^{1} \frac{r_{3}^{1} r_{2}^{1}}{f^{1}}+d^{2} \frac{r_{3}^{2} r_{2}^{2}}{f^{2}}+. ., \ldots \ldots . \\
\ldots \ldots . . & \ldots \ldots \ldots . .
\end{array}\right]
$$

Each column of $\mathbf{H}_{f}$ is itself a sum of subcolumns contributed by each mesh $v$ with a factor $\frac{d^{v}}{f^{v}}$. Hence the $n$-linear determinant splits into a sum of determinants related to the subcolumns; $\mathbf{G}$ is the Gram matrix of a family of $n$ vectors:

$$
\begin{gathered}
\operatorname{det} \mathbf{H}_{f}=\sum_{1 \leq \nu_{1}<. .<v_{n} \leq \nu_{\max }} d^{\nu_{1}} \ldots d^{\nu_{n}} \frac{\operatorname{det} \mathbf{G}\left(\boldsymbol{r}^{\nu_{1}}, \ldots, \boldsymbol{r}^{\nu_{n}}\right)}{f^{\nu_{1}} \ldots f^{\nu_{n}}} \\
\mathbf{G}=\left[g_{i, j}\right] \quad g_{i, j}\left(\boldsymbol{r}^{\nu_{i}}, \ldots, \boldsymbol{r}^{\nu_{j}}\right)=\tilde{\boldsymbol{r}}\left(\boldsymbol{x}^{\nu_{i}}\right) \cdot \tilde{\boldsymbol{r}}\left(\boldsymbol{x}^{\nu_{j}}\right)
\end{gathered}
$$


A Gram determinant is strictly positive except when some $\boldsymbol{r}^{v}$ vanishes. Accordingly, as far as we consider only the meshes effectively observed by one or more detectors, $\operatorname{det} \mathbf{H}_{f}$ is a strictly convex function of $f$ because each term $1 / f^{\nu_{1}} \ldots f^{v_{n}}$ is. As $f$ is constrained by 31 to a compact set, $\operatorname{det} \mathbf{H}_{f}$ reaches a minimum. The latter is characterised by its first order insensitiveness to variations $\delta f$ preserving the total weight: $\sum_{v=1}^{v_{\max }} d^{v} \delta f^{v}=0$.

The variation of $\mathbf{H}_{f+\delta f}=\mathbf{H}_{f}+\delta \mathbf{H}$ due to $\delta f$ is obtained by means of the Eq. (47) with the identities $\frac{1}{f^{v}+\delta f^{v}} \simeq \frac{1}{f^{v}}-\frac{\delta f^{v}}{f^{v} f^{v}}$ and $\frac{\boldsymbol{r}^{v_{k}}}{f^{v}}=\boldsymbol{r}_{f}^{v_{k}}$ :

$$
\begin{aligned}
& \delta h_{i, j}=-\delta f^{1} d^{1} r_{f i}^{1} r_{f j}^{1}-\delta f^{2} d^{2} r_{f i}^{2} r_{f j}^{2}-\ldots \\
& \delta \mathbf{H}=-\sum_{\nu=1}^{\nu_{\max }} d^{\nu} f^{\nu} \boldsymbol{r}_{f}\left(\boldsymbol{x}^{\nu}\right){ }^{t} \boldsymbol{r}_{f}\left(\boldsymbol{x}^{\nu}\right)
\end{aligned}
$$

It is known that $\operatorname{det}\left(\mathbf{H}_{f}+\delta \mathbf{H}\right) \simeq \operatorname{det} \mathbf{H}_{f}\left(1+\operatorname{tr}\left(\mathbf{H}_{f}{ }^{-1} \delta \mathbf{H}\right)\right)$ and it is easily seen that for any two vectors $\boldsymbol{a}, \boldsymbol{b}$ we have: $\operatorname{tr}\left(\mathbf{H}_{f}^{-1} \boldsymbol{a}^{t} \boldsymbol{b}\right)=^{t} \boldsymbol{b} \mathbf{H}_{f}^{-1} \boldsymbol{a}$. This leads finally to the following result:

$$
\delta \operatorname{det} \mathbf{H}_{f}=-\operatorname{det} \mathbf{H}_{f} \sum_{\nu=1}^{\nu_{\max }} d^{\nu} \delta f^{\nu}{ }^{t} \boldsymbol{r}_{f}\left(\boldsymbol{x}^{\nu}\right) \mathbf{H}_{f}^{-1} \boldsymbol{r}_{f}\left(\boldsymbol{x}^{\nu}\right)
$$

The variation 50 vanishes for all admissible $\delta f$ if and only if the ${ }^{t} \boldsymbol{r}_{f}\left(\boldsymbol{x}^{\nu}\right) \mathbf{H}_{f}^{-1} \boldsymbol{r}_{f}\left(\boldsymbol{x}^{v}\right)$ are all equal. This is indeed the constraint 32 .

Acknowledgements. The author is deeply grateful to B. Sportisse to L. Gallardo-Klenner, A. Baklanov, M. Bocquet, A. Ouahsine and G. Roussel for their encouragement, to S. Lacour, G. Adde, H. Cantalloube, F. Lejeune, J. Louchet, V. Mallet and W. Moufouma Okia for their advice. The comments of the referees S. Houweling and C. Rödenbeck were precious.

Edited by: M. Heimann

\section{References}

Anderson, B. D. O. and Moore, J. B.: Optimal filtering, PrenticeHall Information and System Sciences Series, edited by: Kailath, T., Prentice-Hall, Inc., 1979.

Ashbaugh, L. L., Malm, W. C., and Sadeh, W. Z.: A residence time probability analysis of sulfur concentrations at Grand Canyon National park, Atmos. Envir., 19 (8), 1263-1270, 1985.

Baklanov, A.: Modelling of the atmospheric radionuclide transport: local to regional scale, Numerical Mathematics and Mathematical Modelling, INM RAS, Moscow, volume 2, (special issue dedicated to 75-year jubilee of academician G. I. Marchuk), 244 266, 2000

Baklanov, A. and Mahura, A.: Assessment of possible airborne impact from risk sites: methodology for probabilistic atmospheric studies, Atmos. Chem. Phys., 3, 485-495, 2004.

Bennett, A. F. and McIntosh, P. C.: Open ocean modeling as an inverse problem: tidal theory, J. Phys. Oceanogr. 12, 1004-1018, 1982.
Bertero, M., de Mol, C., and Pike, E. R.: Linear inverse problems with discrete data, I: General formulation and singular system analysis, II: Stability and regularisation, Inverse Problems, 1, 301-330, 1985 (part I) and 4, 573-594, 1988 (part II).

Bousquet, P., Peylin, P., Ciais, P., Rayner, P., Friedlingstein, P., Lequere, C., and Tans, P.: Interannual $\mathrm{CO}_{2}$ sources and sinks as deduced by inversion of atmospheric $\mathrm{CO}_{2}$ data, Science, 290, 1342-1346, 2000.

Boutahar, J., Lacour, S., Mallet, V., Quélo, D., Roustan, Y., and Sportisse, B.: Development and validation of a fully modular platform for the numerical modeling of air pollution: POLAIR, Int. J. Envir. Poll., 2003-4.

Bouttier, F. and Courtier, P.: Data assimilation concepts and methods, March 1999, available on the web site: www.ecmwf. int/newsevents/training/rcourse_notes/DATA_ASSIMILATION/ ASSIM_CONCEPTS/Assim_concepts21.html, Meteorological training course lecture series, printed 9 January 2001.

Brandt, J., Ebel, A., Elbern, H., Jakobs, H., Memmesheimer, M., Mikkelsen, T., Thykier-Nielsen, S., and Zlatev, Z.: The importance of accurate meteorological input fields and accurate planetary boundary layer parameterizations, tested against ETEX1, in ETEX symposium on long-range atmospheric transport, model verification and emergency response, 13-16 May 1997, Vienna, Austria, Nodop editor, Proceedings, European Commission, EUR 17346 EN, 195-198, 1997.

Cheney, E. W.: Introduction to approximation theory, International series in pure and applied mathematics, McGraw-Hill, 1966.

Clerbaux, C. , Hadji-Lazaro, J., Turquety, S. , Mégie, G., and Coheur, P.: Trace gas measurements from infrared satellite for chemistry and climate applications, Atmos. Chem. Phys., 3, 1495-1508, 2003,

\section{SRef-ID: 1680-7324/acp/2003-3-1495.}

Courtier, P.: Dual formulation of four-dimensional variational assimilation, Quart. J. Roy. Meteorol. Soc., 123, 2449-2461, 1997.

Enting, I. G., Trudinger, C. M., and Francey, R. J.: A synthesis inversion of the concentration and $\delta^{13} \mathrm{C}$ of atmospheric $\mathrm{CO}_{2}$, Tellus, 47B, 35-52, 1995.

Enting, I. G.: Green's function methods of tracer inversion, in: Inverse methods in global biogeochemical cycles, edited by: Kasibhatla, P., Heimann, M., Rayner, P., Mahowald, N., Prinn, R.G. and Hartley, D.E., Geophysical Monograph Series, American Geophysical Union, Washington DC, 2000.

Fan, S. M., Sarmiento, J. L., Gloor, M., and Pacala, S. W.: On the use of regularization techniques in the inverse modeling of atmospheric carbon dioxide, J. Geophys. Res., 104, 17, 21503 $21512,1999$.

Fano, G.: Metodi matematici della meccanica quantistica, Zanichelli, 1967, translated in English as: Mathematical methods of quantum mechanics, edited by: Landovitz, L. F., McGraw Hill, 1971

Gallardo, L., Olivares, G., Langner, J., and Aarhus, B.: Coastal lows and sulfur air pollution in central Chile, Atmos. Envir., 36, 3829-3841, 2002.

Golub, G. H. and Van Loan, C. F.: Matrix computations, The Johns Hopkins University Press, Baltimore, 1983.

Gram, J. P.: Om Rackkendvilklinger bestemte ved Hjaelp af de minfste Kvadraters Methode, Copenhagen, 1879, translated in German as: Über die Entwicklung reeller Funktionen in Reihen mittels der Methode der kleinsten Quadrate, Journal für reihe und 
angewandte Mathematik, 94, 41-73, 1883.

Hartley, R. V. L.: Transmission of information, Bell System Technical Journal, 7 (3), 535-563, 1928.

Hatori, H.: A note on the entropy of a continuous distribution, Kodai Mathematical Seminar Reports, 10, 172-176, 1958.

Issartel, J.-P. and Baverel, J.: Inverse transport for the verification of the CTBT, Atmos. Chem. Phys., 3, 475-486, 2003,

\section{SRef-ID: 1680-7324/acp/2003-3-475.}

Issartel, J.-P.: Rebuilding sources of linear tracers after atmospheric concentration measurements, Atmos. Chem. Phys., 3, 21112125, 2003, SRef-ID: 1680-7324/acp/2003-3-2111.

Joint Research Centre, Etex. The European tracer experiment, European communities, EUR 18143 EN, ISBN 92-828-5007-2, 107 pp., 1998.

Kholevo, A. S.: Вероятностные и статистические аспекты квантовои теории, Москва, Наука, 1980, translated in English as: Holevo, A. S.: Probabilistic and statistical aspects of quantum theory, North Holland Series in Statistics and Probability, Vol. 1, edited by: Krishnaiah, P. R., Rao, C. R., Rosenblatt, M., and Rozanov, Y.A., North Holland, 1982.

Loren, A. C.: Optimal nonlinear objective analysis, Quart. J. Roy. Meteorol. Soc., 112, 1177-1194, 1988.

Lorensen, W. E. and Cline, H. E.: Marching cubes: a high resolution 3D surface construction algorithm, Computer Graphics, 21, 3, 163-169, 1987.

Louis, J.-F.: A parametric model of vertical eddy fluxes in the atmosphere, Boundary Layer Meteorology, 17, 187-202, 1979.

Louis, J.-F., Tiedke, M., and Geleyn, J.-F.: A short history of the PBL parameterization at ECMWF, Proceedings ECMWF workshop on planetary boundary layer parameterization, 59-79, 1982.

Mallet, V. and Sportisse, B.: 3-D chemistry-transport model Polair: numerical issues, validation and automatic-differentiation strategy, Atmos. Chem. Phys. Discuss., 4, 1371-1392, 2004,

\section{SRef-ID: 1680-7375/acpd/2004-4-1371}

Marchuk, G. I.: Сопряженные Уравнения и Анализ Сложных Систем, Москва, Наука, 1992, translated in English as: Adjoint Equations and Analysis of Complex Systems, Mathematics and its Applications, volume 295, Hazewinkel editor, Kluwer Academic Publisher, 1995.

Marchuk, G. I.: Perturbation theory and the statement of inverse problems. Lecture Notes in Computer Science, 4, 159, 1973.

Marchuk, G. I.: Equation for value of information from meteorological satellites and formulation of inverse problems, Space Res., 2, 3, 462-477, 1964.

Middleton, D.: An Introduction to Statistical Communication Theory, International Series in Pure and Applied Physics, McGraw Hill (see p. 307-310), 1960.

Ouahsine, A. and Smaoui, H.: Flux-limiter schemes for oceanic tracers: application to the English Channel tidal model, Comput. Methods Appl. Mech. Engrg., 179, 307-325, 1999.

Penenko, V. and Baklanov, A.: Methods of sensitivity theory and inverse modeling for estimation of source term and nuclear risk/vulnerability areas, Lecture Notes in Computer Science (LNCS), Springer, 2074, 57-66, 2001.

Penenko, V., Baklanov, A., and Tsvetova, E.: Methods of sensitivity theory and inverse modeling for estimation of source term, Future Generation Computer Systems, 18, 661-671, 2002.

Pudykiewicz, J.: Application of adjoint tracer transport equations for evaluating source parameters, Atmos. Envi., 32 (17), 3039-
3050, 1998.

Quélo, D.: Simulation numérique et assimilation de données variationnelles pour la dispersion atmosphérique de polluants, $\mathrm{PhD}$.Thesis, Ecole Nationale des Ponts et Chaussées, December 8, 2004.

Raschewski, P. K.: Риманова геометрия и тензорнии анализ, Гостехиздат, Москва, 1953, translated in German as: Riemannsche Geometrie und Tensoranalysis, VEB Deutscher Verlag der Wissenschaften, Berlin, 1959.

Rockafellar, R. T.: Convex analysis, Princeton University Press, 1970.

Robertson, L. and Persson, C.: Attempts to Apply Four Dimensional Data Assimilation of Radiological Data Using the Adjoint Technique, Radiation Protection Dosimetry, 50, 333-337, 1993.

Rödenbeck, C., Houweling, S., Gloor, M., and Heimann, M.: $\mathrm{CO}_{2}$ flux history 1982-2001 inferred from atmospheric data using a global inversion of atmospheric transport, Atmos. Chem. Phys. Discuss., 3, 2575-2659, 2003,

SRef-ID: 1680-7375/acpd/2003-3-2575.

Roussel, G., Ternsisien, E., and Benjelloun, M.: Estimation d'un modèle stationnaire de dispersion et localisation de source. Application à la surveillance de la pollution, Revue du Traitement du Signal, 19 (1), 37-48, 2002.

Sartelet, K. N., Boutahar, J., Quélo, D., Coll, I., and Sportisse, B.: Development and validation of a $3 \mathrm{D}$ chemistry transport model POLAIR3D, by comparison with data from ESQUIF campaign, Proc. of the 6th GLOREAM workshop: Global and Regional Atmospheric Modelling, Aveiro, Portugal, September 4-6, 2002.

Seibert, P.: Inverse modelling with a Lagrangian particle dispersion model: application to point releases over limited time intervals, in: Air Pollution and its Application XIV, edited by: Gryning, S.E. and Schiermeier, F.A., 381-389, NATO, Kluwer Academic/Plenum Publisher, 2001.

Shannon, C. E.: A mathematical theory of communication, Bell System Technical Journal, 27 (3), 379-423 and 27 (4), 623-656, 1948.

Sharan, M., McNider, R. T., Gopalakrishnan, S. G., and Singh, M. P.: Bhopal gas leak: a numerical simulation of episodic dispersion, Atmos. Envir., 29, 2051-2059, 1995.

Sportisse, B., Boutahar, J., Debry, E., Quélo, D., and Sartelet, K.: Some tracks in air pollution modeling and simulation, Revista de la Real Academia de Ciencias, Serie A: Matemáticas (RACSAM), 96 (3), 507-528, 2002.

Stohl, A.: Computation, accuracy and applications of trajectories a review and bibliography, Atmos. Envir., 32 (6), 947-966 (see p. 959), 1998.

Tarantola, A.: Inverse problem theory, Elsevier, 1987.

Uliasz, M. and Pielke, R.: Application of the receptor oriented approach in mesoscale dispersion modeling, in van Dop and Steyn editors, Air Pollution and its Application VIII, 399-407, NATO, Plenum Publisher, 1991.

Wendum, D.: Three long-range transport models compared to the ETEX experiment: a performance study, Atmos. Envir., 32 (24), 4297-4305, 1998.

Wotawa, G., de Geer, L.-E., Denier, P., Kalinowski, M., Toivonen, H., d'Amours, R., Desiato, F., Issartel, J.-P., Langer, M., Seibert, P., Frank, A., Sloan, C., and Yamazawa, H.: Atmospheric transport modelling in support of CTBT verification - overview and basic concepts, Atmos. Envir., 37, 2525-2537, 2003. 